\title{
Comparison between Finite-Difference Time- Domain Method and Experimental Results for Femtosecond Laser Pulse Propagation
}

\author{
Shinki Nakamura \\ Ibaraki University \\ Japan
}

\section{Introduction}

There has recently been significant interest in the generation of single-cycle optical pulses by optical pulse compression of ultrabroad-band light produced in fibers. There have been some experiments reported on ultrabroad-band pulse generation using a silica fiber (Nakamura et al, 2002a), (Karasawa et al, 2000) and an Ar-gas filled hollow fiber (Karasawa et al, 2001) , and the optical pulse compression by nonlinear chirp compensation (Nakamura et al, 2002a), (Karasawa et al, 2001). For these experiments on generating few-optical-cycle pulses, characterizing the spectral phase of ultrabroad-band pulses analytically as well as experimentally is highly important.

Conventionally, the slowly varying-envelope approximation (SVEA) in the beam propagation method (BPM) has been used to describe the propagation of an optical pulse in a fiber (Agrawal, 1995). However, if the pulse duration approaches the optical cycle regime (<10 fs), this approximation becomes invalid (Agrawal, 1995). It is necessary to use the finite-difference time-domain (FDTD) method (Joseph \& Taflove, 1997), (Kalosha \& Herrmann, 2000) without SVEA (Agrawal, 1995). Previous reports by Goorjian (Goorjian et al., 1992), (Joseph et al., 1993), Joseph (Joseph \& Taflove, 1997), (Goorjian et al., 1992), (Joseph et al., 1993), Taflove (Joseph \& Taflove, 1997), (Goorjian et al., 1992), (Joseph et al., 1993), (Taflove \& Hagness., 2000) and Hagness (Goorjian et al., 1992), (Taflove \& Hagness., 2000) (JGTH) proposed an excellent FDTD algorithm considering a combination of linear dispersion with one resonant frequency and nonlinear terms with a Raman response function.

We performed an experiment of chirped $12 \mathrm{fs}$ optical pulse propagation as described in Section 3. In order to compare FDTD calculation results with the experimentally measured ultrabroad-band spectra of such an ultrashort laser pulse, we extend the JGTH algorithm to that considering all of the exact Sellmeier fitting values for ultrabroad-band spectra. Because of the broad spectrum of pulses propagating in a fiber, it becomes much more important to take the accurate linear dispersion into account. It is well known that at least two resonant frequencies are required for the linear dispersion to fit accurately to the refractive index data. A recent report by Kalosha and Herrmann considers the linear dispersion with two resonant frequencies and the nonlinear terms without the Raman effect (Kalosha \& Herrmann, 2000). For the single-cycle pulse generation experiment, we must use at least the shortest pulse of 3.4 fs (Yamane et al., 2003) or sub-5 fs (Karasawa et al, 2001), (Cheng et al., 1998) or the commercially available $12 \mathrm{fs}$ pulses. Such a time regime is comparable to the 
Raman characteristic time of 5 fs (Agrawal, 1995) in a silica fiber. Therefore, it is very important to consider not only the accurate linear dispersion of silica but also the Raman effect in a silica fiber in the few-optical-cycles regime. In addition, because of the high repetition rate and pulse intensity stability, in particular, ultrabroad-band supercontinuum light generation and few-optical-cycles pulse generation by nonlinear pulse propagation in photonic crystal fibers (Ranka et al., 2000) and tapered fibers (Birks et al., 2000), which are both made of silica, have attracted much attention. In this work, we have extended the FDTD method with nonlinear polarization $P_{\mathrm{NL}}$ involving the Raman response function (JGTH-algorithm) to $12 \mathrm{fs}$ ultrabroadband pulse propagation in a silica fiber with the consideration of linear polarization $\mathrm{P}_{\mathrm{L}}$, including all exact Sellmeier-fitting values of silica with three resonant frequencies, in order to compare the calculation results with our experimental results (Nakamura et al, 2002a), (Karasawa et al, 2000). We have already compared the extended FDTD method (Nakamura et al., 2002b) with BPM by applying the split-step Fourier (SSF) method which is the solution of a generalized nonlinear Schrödinger equation (GNLSE) with SVEA(Agrawal, 1995), and the extended FDTD agreed better with the experimental results than with BPM. However, we have not shown the details of the calculation algorithm and temporal characteristics, and did not consider a chirp of the initial incident pulse to a fiber. In this chapter, we show the details of the the extended FDTD calculation algorithm (Nakamura et al., 2002c), (Nakamura et al., 2003) temporal characteristics of the pulse, and consider a chirp of the initial incident pulse to a fiber, and finally, we demonstrate the group delay compensation which generates the compressed pulse. Since 2004, the extended FDFD is called as the auxiliary differential Equation (ADE)FDTD (Fujii et al., 2004). Additionally, we compared between the extended FDTD calculation and experimental result in dual wavelengths pulses propagation in a fiber. Finally, we investigated the slowly varying envelope approximation breakdown by comparing between BPM and the extended FDTD numerical results.

\section{Extended FDTD algorithm}

For simplicity, the electric and magnetic fields are expressed by Ey and $H x$ and onedimensional propagation along the $z$ direction is considered. The optical fiber is assumed to be isotropic and nonmagnetic. If a linear configuration is assumed, Maxwell's equations are as follows:

$$
\begin{aligned}
& \frac{\partial H_{x}}{\partial t}=\frac{1}{\mu_{0}} \frac{\partial E_{y}}{\partial z}, \\
& \frac{\partial D_{y}}{\partial t}=\frac{\partial H_{x}}{\partial z},
\end{aligned}
$$

where $\mu_{0}$ is the permeability in a vacuum and $D y$ is the dielectric flux density. By means of Yee's central difference method, Eq. (1) can be expressed by the following, in which the time and spatial steps are shifted by $1 / 2$ step:

$$
\begin{aligned}
& \left.H_{x}\right|_{i+1 / 2} ^{n+1 / 2}=\left.H_{x}\right|_{i+1 / 2} ^{n-1 / 2}+\frac{\Delta t}{\mu_{0} \Delta z}\left(\left.E_{y}\right|_{i+1} ^{n}-\left.E_{y}\right|_{i} ^{n}\right), \\
& \left.D_{y}\right|_{i} ^{n+1}=\left.D_{y}\right|_{i} ^{n}+\frac{\Delta t}{\Delta z}\left(\left.H_{x}\right|_{i+1 / 2} ^{n+1 / 2}-\left.H_{x}\right|_{i-1 / 2} ^{n+1 / 2}\right),
\end{aligned}
$$


where $n$ is the time step number and $i$ is the spatial step number. They are $t=n \Delta t$ and $z=$ $i \Delta z$, respectively.

As the third step, let us use

$$
D(\omega)=\varepsilon_{0} \varepsilon_{r}(\omega) E(\omega)
$$

to derive a new $\left.E_{y}\right|_{i} ^{n+1}$. Then, the iteration algorithm of the FDTD is complete. The authors have introduced the linear polarization $P_{L}$ and the nonlinear polarization $P_{N L}$, corresponding to the Sellmeier fitting equation into the third step above, at the same time. First, the total polarization is expressed as

$$
\begin{aligned}
P & =\varepsilon_{0}\left(\chi^{(1)} E+\chi^{(2)} E^{2}+\chi^{(3)} E^{3}+\cdots\right) \\
& =P_{L}+P_{N L} .
\end{aligned}
$$

For simplicity, only the linear polarization is considered at first. The linear polarization is expressed as

$$
\begin{gathered}
P_{L}=\sum_{i=1}^{3} P_{i}, \\
P_{i}=\varepsilon_{0} \int_{0}^{t} \chi_{i}^{(1)}(t-\tau) E(\tau) d \tau,
\end{gathered}
$$

where $\chi_{i}^{(1)}$ has a Lorenz form with respect to frequency and is described as follows (Karasawa et al, 2001), (Agrawal, 1995):

$$
\chi_{i}^{(1)}(\omega)=\frac{G_{i} \omega_{i}^{2}\left(\varepsilon_{s}-1\right)}{\omega_{i}^{2}+2 j \omega \delta_{i}^{2}-\omega^{2}},(i=1,2,3) .
$$

Here, $j$ is the imaginary unit, $\varepsilon_{s}$ is the permittivity in an electrostatic field, $\omega_{i}$ is the resonant angular frequency, $\delta_{i}$ is the attenuation constant with respect to resonant absorption, and $\sum_{i=1}^{3} G_{i}=1$. If the above is inversely Fourier-transformed by assuming that $\omega_{i}$ is constant, the following differential equation is obtained:

$$
\left[\chi_{i}^{(1)}\right]^{\prime \prime}+2 \delta_{i}\left[\chi_{i}^{(1)}\right]^{\prime}+\omega_{i}^{2} \chi_{i}^{(1)}=0
$$

where $\chi_{i}^{(1)}(t=0)=0$ and $\left[\chi_{i}^{(1)}\right]^{\prime}(t=0)=G_{i} \omega_{i}^{2}\left(\varepsilon_{s}-1\right)$. If the optical fiber is sufficiently short that the attenuation term $\delta_{i}$ due to absorption can be neglected, then $\delta_{i}=0$, so that Eq. (7) can be described as follows:

$$
\chi_{i}^{(1)}(\omega)=\frac{G_{i}\left(\varepsilon_{s}-1\right) \cdot \omega_{i}^{2}}{\omega_{i}^{2}-\omega^{2}},(i=1,2,3) .
$$


Then, $\varepsilon_{r}(\omega)=1+\sum_{i=1}^{3} \chi_{i}^{(1)}(\omega)$ and the Sellmeier equation (Nakamura et al, 2002) is

$$
\varepsilon_{r}(\omega)=n(\omega)^{2}=1+\sum_{i=1}^{3} \frac{b_{i} \cdot \omega_{i}^{2}}{\omega_{i}^{2}-\omega^{2}} .
$$

Therefore,

$$
\chi_{i}^{(1)}(\omega)=\frac{G_{i}\left(\varepsilon_{s}-1\right) \cdot \omega_{i}^{2}}{\omega_{i}^{2}-\omega^{2}}=\frac{b_{i} \cdot \omega_{i}^{2}}{\omega_{i}^{2}-\omega^{2}}
$$

where $b_{i}=G_{i}\left(\varepsilon_{s}-1\right)$. Also, since $\sum_{i=1}^{3} b_{i}=\sum_{i=1}^{3} G_{i}\left(\varepsilon_{s}-1\right)=\left(\varepsilon_{s}-1\right) \sum_{i=1}^{3} G_{i}=\left(\varepsilon_{s}-1\right)$, we have

$$
\varepsilon_{s}=1+\sum_{i=1}^{3} b_{i}
$$

The derivation above is summarized as follows:

$$
\begin{aligned}
D(\omega) & =\varepsilon_{0} E(\omega)+P(\omega) \\
& =\varepsilon_{0}\left(1+\sum_{i=1}^{3} \frac{b_{i} \cdot \omega_{i}^{2}}{\omega_{i}^{2}-\omega^{2}}\right) E(\omega) \\
& =\varepsilon_{0} E(\omega)+\varepsilon_{0}\left(\sum_{i=1}^{3} \frac{b_{i} \cdot \omega_{i}^{2}}{\omega_{i}^{2}-\omega^{2}}\right) E(\omega) .
\end{aligned}
$$

If the nonlinear polarization is taken into account by returning to Eq. (4), then Eq. (13) can be treated as follows:

$$
E=\frac{1}{\varepsilon_{0}}\left(D-\sum_{i=1}^{3} P_{i}-P_{N L}\right)
$$

where

$$
P_{N L}=\varepsilon_{0} \chi^{(3)} E \int_{-\infty}^{\infty}\left[\alpha \delta(t-\tau)+(1-\alpha) g_{R}(t-\tau)\right][E(\tau)]^{2} d \tau
$$

Here, $\delta(t-\tau)$ is the delta function and $\alpha$ is the ratio of the intensities of the Kerr effect and the Raman effect. Also, $g_{R}(t)=\left[\left(\tau_{1}^{2}+\tau_{2}^{2}\right) / \tau_{1} \tau_{2}^{2}\right] e^{-t / \tau_{2}} \sin \left(t / \tau_{1}\right)$. When Eqs. (11) and (12) are taken into consideration in Eqs. (14) and (15), we obtain

$$
P_{i}^{\prime \prime}+\omega_{i}^{2} P_{i}=\omega_{i}^{2} b_{i}\left(D-\sum_{i=1}^{3} P_{i}-P_{N L}\right) .
$$

Further, if the nonlinear polarization $P_{N L}$ in Eq. (15) is described as 


$$
P_{N L}=\chi^{(3)} E(t)\left[\varepsilon_{0} \alpha E(t)^{2}+(1-\alpha) G\right], \text { then }
$$

$$
G=\varepsilon_{0} \int_{-\infty}^{\infty} g_{R}(t-\tau)[E(\tau)]^{2} d \tau .
$$

Also, if Eq. (16) is replaced by $G$, we obtain

$$
P_{i}^{\prime \prime}+\omega_{i}^{2} P_{i}=\omega_{i}^{2} b_{i}\left(D-\sum_{i=1}^{3} P_{i}-\chi^{(3)}(1-\alpha) E \cdot G-\varepsilon_{0} \chi^{(3)} \alpha E^{3}\right)
$$

leading to the form

$$
\begin{aligned}
& P_{1}^{\prime \prime}+\omega_{1}^{2}\left(1+b_{1}\right) P_{1}+\omega_{1}^{2} b_{1} P_{2}+\omega_{1}^{2} b_{1} P_{3} \\
& +\omega_{1}^{2} b_{1} \chi^{(3)} \cdot\left[(1-\alpha) E G+\varepsilon_{0} \alpha E^{3}\right]=\omega_{1}^{2} b_{1} D \\
& P_{2}^{\prime \prime}+\omega_{2}^{2}\left(1+b_{2}\right) P_{2}+\omega_{2}^{2} b_{2} P_{1}+\omega_{2}^{2} b_{2} P_{3} \\
& +\omega_{2}^{2} b_{2} \chi^{(3)} \cdot\left[(1-\alpha) E G+\varepsilon_{0} \alpha E^{3}\right]=\omega_{2}^{2} b_{2} D \\
& P_{3}^{\prime \prime}+\omega_{3}^{2}\left(1+b_{3}\right) P_{3}+\omega_{3}^{2} b_{3} P_{1}+\omega_{3}^{2} b_{3} P_{2} \\
& +\omega_{3}^{2} b_{3} \chi^{(3)} \cdot\left[(1-\alpha) E G+\varepsilon_{0} \alpha E^{3}\right]=\omega_{3}^{2} b_{3} D .
\end{aligned}
$$

Further, applying a central difference scheme centered at time step $n$, these equations yield

$$
\begin{aligned}
& \left.a_{1} P_{1}\right|^{n+1}+\left.c_{1} P_{2}\right|^{n+1}+\left.c_{1} P_{3}\right|^{n+1} \\
& =c_{1}\left(\left.D\right|^{n+1}+\left.D\right|^{n-1}\right)-c_{1}\left(\left.P_{2}\right|^{n-1}+\left.P_{3}\right|^{n-1}\right) \\
& +\left.4 P_{1}\right|^{n}+\left.g_{1} P_{1}\right|^{n-1}-\omega_{1}^{2} b_{1} \chi^{(3)} \cdot\left[\left.\left.(1-\alpha) E\right|^{n} G\right|^{n}+\varepsilon_{0} \alpha\left(\left.E\right|^{n}\right)^{3}\right] \\
& \left.c_{2} P_{1}\right|^{n+1}+\left.a_{2} P_{2}\right|^{n+1}+\left.c_{2} P_{3}\right|^{n+1} \\
& =c_{2}\left(\left.D\right|^{n+1}+\left.D\right|^{n-1}\right)-c_{2}\left(\left.P_{1}\right|^{n-1}+\left.P_{3}\right|^{n-1}\right) \\
& +\left.4 P_{2}\right|^{n}+\left.g_{2} P_{2}\right|^{n-1}-\omega_{2}^{2} b_{2} \chi^{(3)} \cdot\left[\left.\left.(1-\alpha) E\right|^{n} G\right|^{n}+\varepsilon_{0} \alpha\left(\left.E\right|^{n}\right)^{3}\right] \\
& \left.c_{3} P_{1}\right|^{n+1}+\left.c_{3} P_{2}\right|^{n+1}+\left.a_{3} P_{3}\right|^{n+1} \\
& =c_{3}\left(\left.D\right|^{n+1}+\left.D\right|^{n-1}\right)-c_{3}\left(\left.P_{1}\right|^{n-1}+\left.P_{2}\right|^{n-1}\right) \\
& +\left.4 P_{3}\right|^{n}+\left.g_{3} P_{3}\right|^{n-1}-\omega_{3}^{2} b_{3} \chi^{(3)} \cdot\left[\left.\left.(1-\alpha) E\right|^{n} G\right|^{n}+\varepsilon_{0} \alpha\left(\left.E\right|^{n}\right)^{3}\right],
\end{aligned}
$$

Where 


$$
\begin{gathered}
a_{i}=2+\omega_{i}^{2} \Delta t^{2}\left(1+b_{i}\right) \\
c_{i}=\omega_{i}^{2} \Delta t^{2} b_{i} \\
g_{i}=-2-\omega_{i}^{2} \Delta t^{2}\left(1+b_{i}\right) .
\end{gathered}
$$

Here, $\left.E\right|^{n}$ and $\left.G\right|^{n}$ have already been updated in a previous time step. From the above, the unknown $\left.P_{1}\right|^{n+1},\left.P_{2}\right|^{n+1}$ and $\left.P_{3}\right|^{n+1}$ can be derived. Here, by using

$$
\begin{aligned}
F=P_{L} & =\sum_{i=1}^{3} P_{i} \\
& =\sum_{i=1}^{3} \varepsilon_{0} \int_{0}^{t} \chi_{i}^{(1)}(t-\tau) E(\tau) d \tau,
\end{aligned}
$$

the linear polarization $P_{L}$ is replaced by $P_{L}=F$. Then,

$$
\left.F\right|^{n+1}=\left.P_{L}\right|^{n+1}=\left.P_{1}\right|^{n+1}+\left.P_{2}\right|^{n+1}+\left.P_{3}\right|^{n+1}
$$

is explicitly obtained. Hence, an unknown linear polarization can be found. The reason why only one resonant frequency can be taken into account for the linear polarization in the JGTH algorithm (Joseph \& Taflove, 1997), (Goorjian et al., 1992), (Joseph et al., 1993) (Taflove \& Hagness., 2000) is as follows. Since we take $G$ as $\left.G\right|^{n}$ in applying the central difference to Eq. (19) to obtain Eq. (20), we can obtain the linear polarization explicitly. However $\left(\left.G\right|^{n+1}+\left.G\right|^{n-1}\right) / 2$ was used in the previous JGTH algorithm to improve the finite-difference accuracy. In this case, the unknown $\left.G\right|^{n+1}$ is necessary, and therefore the successive solution of the nonlinear polarization after the linear polarization is derived is not possible algorithmically. Therefore, in the JGTH algorithm, the linear polarization is restricted to one so that symmetry is provided to the differential equations for linear polarization and nonlinear polarization. This is followed by the solution of the simultaneous finite difference equations to obtain two unknowns simultaneously. Since only the known $\left.G\right|^{n}$ is used by our method in the finite difference equation for the linear polarization, it is possible to derive the nonlinear polarization after the linear polarization is found. The accuracy of the finite difference method is sufficient in this procedure. As described in detail below, the use of only one resonant frequency for the linear polarization when the results are compared with experimental results is a serious problem due to its more significant approximation of dispersion, as follows.

Let us now describe the approximation of the dispersion in the conventional FDTD method. The equation used in Refs. (Joseph \& Taflove, 1997)-(Taflove \& Hagness., 2000), in a series of conventional FDTD methods, called the JGTH algorithm, is the case of $i=1$ in Eq. (7) and $\varepsilon_{r}(\omega)=n(\omega)^{2}=\varepsilon_{\infty}+b_{1} \omega_{1} /\left(\omega_{1}^{2}{ }^{2}-\omega^{2}\right)$ in Eq. (10). Here, $\varepsilon_{\infty}$ is the relative permittivity when the frequency is infinite. It is not possible in principle to approximate the refractive index data of fused silica in the entire frequency range in this equation. However, it is possible within a limited frequency range. For example, if an approximation is introduced such that the error of the refractive index is $0.1 \%$ at $250-517 \mathrm{THz}$, then the parameters can be determined as $\varepsilon_{\infty}=$ 
$0.9560, b_{1}=1.130$ and $\omega_{1}=1.1639 \times 10^{16} \mathrm{rad} / \mathrm{s}$. Using these values, the values of $\beta_{1}, \beta_{2}$ and $\beta_{3}$ are found to be $4.901 \times 10^{-9} \mathrm{~s} / \mathrm{m}, 7.287 \times 10^{-26} \mathrm{~s}^{2} / \mathrm{m}$ and $3.469 \times 10^{-41} \mathrm{~s}^{3} / \mathrm{m}$, respectively. $\beta_{n}(\mathrm{n}=1,2,3, \cdots)$ is the $n$-th order derivative of propagation constant $\beta_{0}$ at an incident center angular frequency $\omega_{0}$ defined as $\beta_{n}=\left(d^{n} \beta / d \omega^{n}\right)_{\omega=\omega_{0}}$ (Agrawal, 1995). In contrast, the Sellmeier equation with three resonant frequencies used in the extended FDTD method by the authors accurately approximates the refractive index in the entire frequency range where measurement is possible (Malitson., 1961). If this equation is used, $\beta_{1}=4.894 \times 10^{-9}$ $\mathrm{s} / \mathrm{m}, \beta_{2}=3.616 \times 10^{-26} \mathrm{~s}^{2} / \mathrm{m}$ and $\beta_{3}=2.750 \times 10^{-41} \mathrm{~s}^{3} / \mathrm{m}$. Since the conventional method and the extended FDTD method approximately agree with regard to $\beta_{1}$, the results for the group velocity $v_{g}=\left(\beta_{1}\right)^{-1}$ can be relied upon to a certain extent in the conventional method (Joseph \& Taflove, 1997), (Taflove \& Hagness., 2000). This implies that the conventional method can be used for the calculation of the propagation of a pulse of nanosecond order, for which the spectral width is sufficiently narrow and the effect of the group velocity dispersion is negligible. However, since the group velocity dispersion $\beta_{2}=\partial\left(v_{g}\right)^{-1} / \partial \omega$ differs from the value obtained from the refractive index data by a factor of 2 , the comparison of the conventional method with the propagation experiment of a pulse of sub-nanosecond order or shorter is not possible. Therefore, in the conventional FDTD method of the JGTH algorithm (Joseph \& Taflove, 1997) (Goorjian et al., 1992), (Taflove \& Hagness., 2000), calculation of the propagation of femtosecond pulses can be carried out only for a hypothetical medium after the dispersion value is set to an easily calculated value and $\chi^{(3)}$ is set to an unrealistic value that is $10^{20}$ times the usual value. The comparison between simple JGTH algorithm with parameters of $\varepsilon_{\infty}=0.9560, b_{1}=1.130$ and $\omega_{1}=1.1639 \times 10^{16} \mathrm{rad} / \mathrm{s}$ and the extended FDTD method is shown as temporal and spectral characteristics in section IV.

For the above reason, the nonlinear polarization is derived next from the linear polarization $\left.F\right|^{n+1}$ derived from Eq. (23). The definition of the nonlinear polarization has already been presented in Eq. (15). When Eq. (15) is Fourier-transformed, we obtain

$$
\begin{gathered}
\tilde{P}_{N L}(\omega)=\varepsilon_{0} \chi_{0}^{(3)} \tilde{E}(\omega) \times\left\{\alpha \tilde{E}^{2}(\omega)+(1-\alpha) \tilde{g}_{R} \tilde{E}^{2}(\omega)\right\} \\
\tilde{g}_{R}(\omega)=\frac{\bar{\omega}_{0}^{2}}{\bar{\omega}_{0}^{2}-j \omega \bar{\delta}-\omega^{2}}
\end{gathered}
$$

where $\bar{\delta}=1 / \tau_{2}$ and $\bar{\omega}_{0}^{2}=\left(1 / \tau_{1}\right)^{2}+\left(1 / \tau_{2}\right)^{2}$. Next, if Eq. (17), the definition of $G$, is Fouriertransformed, we obtain

$$
\tilde{G}(\omega)=\varepsilon_{0} \tilde{g}_{R}(\omega) \tilde{E}^{2}(\omega) .
$$

When this is used, Eq. (25) becomes

$$
\left(\bar{\omega}_{0}^{2}-j \omega \bar{\delta}-\omega^{2}\right) \tilde{G}(\omega)=\varepsilon_{0} \bar{\omega}_{0}^{2} \tilde{E}^{2}(\omega) .
$$

Hence, the equation to be solved is expressed as follows by taking the inverse Fourier transform of Eq. (27): 


$$
\frac{1}{\bar{\omega}_{0}^{2}} \frac{\partial^{2} G}{\partial t^{2}}+\frac{\bar{\delta}}{\bar{\omega}_{0}^{2}} \frac{\partial G}{\partial t}+G-\varepsilon_{0} E^{2}=0 .
$$

From the relationship between the dielectric flux density and the electric field,

$$
\begin{aligned}
\tilde{D} & =\varepsilon_{0} \tilde{E}+\tilde{P} \\
& =\varepsilon_{0} \tilde{E}+\tilde{P}_{L}+\tilde{P}_{N L} \\
& =\varepsilon_{0} \tilde{E}+\varepsilon_{0} \chi^{(1)} \tilde{E}+\varepsilon_{0} \chi^{(3)} \tilde{E}\left[\alpha \tilde{E}^{2}+(1-\alpha) \tilde{g}_{R} \tilde{E}^{2}\right] \\
& =\varepsilon_{0}\left(1+\alpha \chi^{(3)} \tilde{E}^{2}\right) \tilde{E}+\tilde{F}+(1-\alpha) \chi^{(3)} \tilde{E} \tilde{G} .
\end{aligned}
$$

Eq. (28) can be modified to the following:

$$
\begin{aligned}
\frac{1}{\bar{\omega}_{0}^{2}} \frac{\partial^{2} G}{\partial t^{2}}+ & \frac{\bar{\delta}}{\bar{\omega}_{0}^{2}} \frac{\partial G}{\partial t} \\
& +\left[1+\frac{(1-\alpha) \chi^{(3)}\left(E_{y}\right)^{2}}{1+\alpha \chi^{(3)}\left(E_{y}\right)^{2}}\right] \cdot G \\
& +\left[\frac{E_{y}}{1+\alpha \chi^{(3)}\left(E_{y}\right)^{2}}\right] \cdot F \\
& =\left[\frac{E_{y}}{1+\alpha \chi^{(3)}\left(E_{y}\right)^{2}}\right] \cdot D_{y} .
\end{aligned}
$$

Further, applying a central difference scheme centered at time step $n$, these equations yield

$$
\begin{gathered}
\left(\frac{1}{\bar{\omega}_{0}^{2} \Delta t^{2}}\right) \cdot\left(\left.G\right|_{i} ^{n+1}-\left.2 G\right|_{i} ^{n}+\left.G\right|_{i} ^{n-1}\right)+\left(\frac{\bar{\delta}}{\bar{\omega}_{0}^{2} \Delta t}\right) \cdot\left(\left.G\right|_{i} ^{n+1}-\left.G\right|_{i} ^{n-1}\right) \\
+\left[\begin{array}{c}
\left.1+\frac{(1-\alpha) \chi^{(3)}\left(\left.E_{y}\right|_{i} ^{n}\right)^{2}}{1+\alpha \chi^{(3)}\left(\left.E_{y}\right|_{i} ^{n}\right)^{2}}\right] \cdot \frac{\left(\left.G\right|_{i} ^{n+1}+\left.G\right|_{i} ^{n-1}\right)}{2} \\
+\left[\frac{\left.E_{y}\right|_{i} ^{n}}{1+\alpha \chi^{(3)}\left(\left.E_{y}\right|_{i} ^{n}\right)^{2}}\right] \cdot \frac{\left(\left.F\right|_{i} ^{n+1}+\left.F\right|_{i} ^{n-1}\right)}{2} \\
=\left[\frac{\left.E_{y}\right|_{i} ^{n}}{1+\alpha \chi^{(3)}\left(\left.E_{y}\right|_{i} ^{n}\right)^{2}}\right] \cdot \frac{\left(\left.D_{y}\right|_{i} ^{n+1}+\left.D_{y}\right|_{i} ^{n-1}\right)}{2}
\end{array} .\right.
\end{gathered}
$$


When $\left.F\right|^{n+1}$ derived from Eq. (23) is substituted into Eq. (31) together with the known $\left.F\right|^{n-1},\left.G\right|^{n},\left.G\right|^{n-1}$ and $\left.E\right|^{n}$, the unknown $\left.G\right|^{n+1}$ can be derived. Here, an explicit form of updating $G$ is presented as

$$
\begin{aligned}
\left.G\right|_{i} ^{n+1}=[ & d+e+f]^{-1} \\
& \times\left\{\left.2 d \cdot G\right|_{i} ^{n}+\left.\left(e-d+\frac{f}{2}\right) \cdot G\right|_{i} ^{n-1}-g \cdot \frac{\left(\left.F\right|_{i} ^{n+1}+\left.F\right|_{i} ^{n-1}\right)}{2}+g \cdot \frac{\left(\left.D_{y}\right|_{i} ^{n+1}+\left.D_{y}\right|_{i} ^{n-1}\right)}{2}\right\} .
\end{aligned}
$$

where

$$
\begin{aligned}
& d=\left(\frac{1}{\bar{\omega}_{0}^{2} \Delta t^{2}}\right) \\
& e=\left(\frac{\bar{\delta}}{\bar{\omega}_{0}^{2} \Delta t}\right) \\
& f=\left[1+\frac{(1-\alpha) \chi^{(3)}\left(\left.E_{y}\right|_{i} ^{n}\right)^{2}}{1+\alpha \chi^{(3)}\left(\left.E_{y}\right|_{i} ^{n}\right)^{2}}\right] \\
& g=\left[\frac{\left.\left.E_{y}\right|_{i} ^{n}\right]}{1+\alpha \chi^{(3)}\left(\left.E_{y}\right|_{i} ^{n}\right)^{2}}\right]
\end{aligned}
$$

When the obtained $\left.G\right|^{n+1}$ and $\left.F\right|^{n+1}$ are substituted into the following, the unknown $\left.E\right|^{n+1}$ can be derived and the iteration algorithm for the FDTD is terminated. Hence,

$$
\begin{aligned}
\left.E_{y}^{p+1}\right|^{n+1} & =\frac{\left.D_{y}\right|^{n+1}-\left.F\right|^{n+1}-\left.\left.(1-\alpha) \chi_{0}^{(3)} E_{y}^{p}\right|^{n+1} G\right|^{n+1}}{\varepsilon_{0}\left[1+\alpha \chi_{0}^{(3)}\left(E_{y}^{p}\right)^{2}\right]} . \\
p & =0,1,2, \cdots
\end{aligned}
$$

Note that the initial condition is $\left.E_{y}^{0}\right|^{n+1}=\left.E_{y}\right|^{n}$. Here, Eq. (34) is derived from Eq. (29).

\section{Femtosecond single pulse propagation}

\subsection{Experimental Setup}

Figure 1 shows the setup used for the authors' experiments (Nakamura et al, 2002a), (Karasawa et al, 2000). The $12 \mathrm{fs}$ and $10 \mathrm{~nJ}$ pulses $\left(\mathrm{sech}^{2}\right)$ at the center wavelength of $800 \mathrm{~nm}$ were generated from a mode-locked Ti:sapphire laser (Femtolaser $\mathrm{GmbH}$, Femtosource M- 
1). The incident pulse width was measured by a fringe-resolved autocorrelator (FRAC). The $12 \mathrm{fs}$ pulses were coupled into a $2.5 \mathrm{~mm}$ silica fiber by a 36x reflective objective (Ealing). The advantage of this kind of reflective objective is that no additional group-delay dispersion (GDD) or third-order dispersion (TOD) is introduced to the pulses. However, the $12 \mathrm{fs}$ pulses are slightly chirped because the pulse width of the full width at half maximum (FWHM) resulting from the Fourier transformation of the fiber input spectrum is $8.5 \mathrm{fs}$.

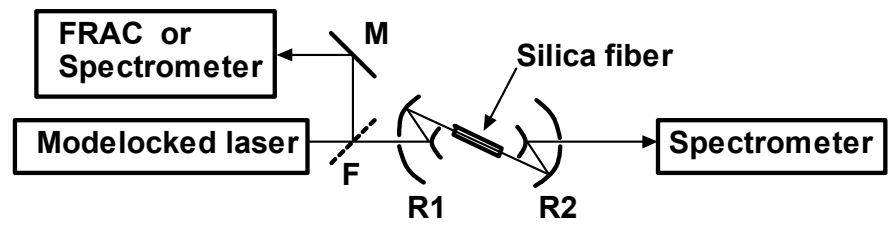

Fig. 1. Layout of the experimental setup (Nakamura et al., 2002a). M, silvercoated plane mirrors; F, flipper beam splitter, R1, R2, reflective objectives (36x); FRAC, fringe resolved autocorrelator.

The input pulse is obtained by calculating the inverse Fourier transform of the spectrum of the input pulse. However, the transform-limited pulse width $(8.5 \mathrm{fs})$ is smaller than the experimentally measured pulse width (12 fs) obtained by a fringe-resolved autocorrelator. To use the pulse with the correct spectrum and the pulse width in the calculation, the temporal duration is adjusted by adding the quadratic spectral phase in the form $\varphi(\omega)=c\left(\omega-\omega_{0}\right)^{2}$ to the experimental spectrum, to render the inverse Fourier transform pulse width the same as that of the measured one. This gave the values of $c=-9.043 \mathrm{fs}^{2} / \mathrm{rad}$ and $\partial^{2} \varphi(\omega) /\left.\partial \omega^{2}\right|_{\omega=\omega_{0}}=-113.6 \mathrm{fs}^{2}$. The peak power of an input pulse was $175 \mathrm{~kW}$. A singlemode fused silica fiber (Newport F-SPV) with a core diameter of $2.64 \mu \mathrm{m}$ was used. The output from the $2.5 \mathrm{~mm}$ fiber was collimated by another reflective objective and measured by a spectrometer. The obtained input and output spectra of the fiber are shown in Fig. 2.

The dashed line in Figure 2 shows the input spectrum and the solid line shows the output spectrum. The dashed line and short-dashed line in Fig. 3 show the assumption of an input spectrum whose form is a sech ${ }^{2}$ shape, using this as field excitations in the extended FDTD calculation (dashed line) and the simpler JGTH-FDTD calculation (short-dashed line). There is no difference between the spectra of the extended FDTD and JGTH-FDTD. The solid line in Fig. 3 shows the experimentally obtained input pulse spectrum. Note that the intensity at

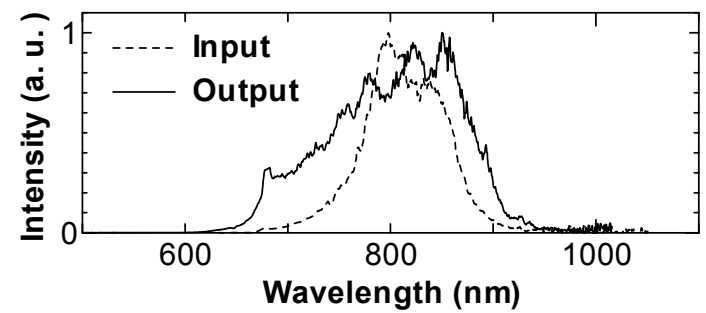

Fig. 2. Input and output spectra measured in the $12 \mathrm{fs}$ laser propagation experiment in a silica fiber (Karasawa et al, 2000). Dashed line shows the input spectrum and solid line shows the output spectrum. 


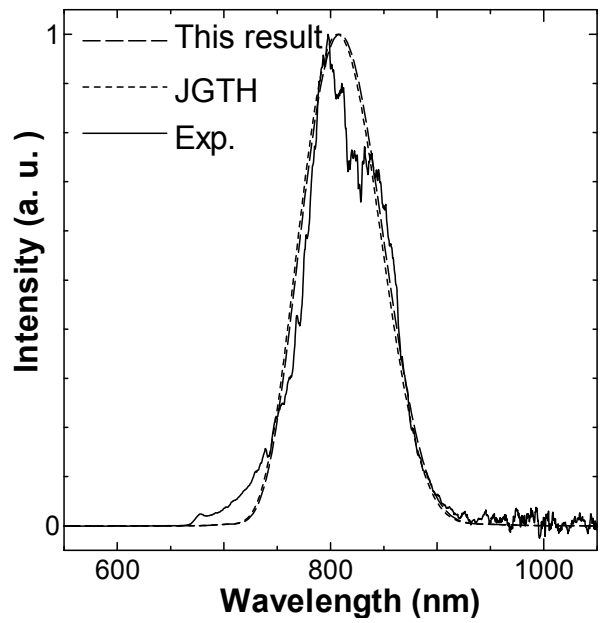

Fig. 3. Spectra of $12 \mathrm{fs}$ incident laser pulse into a $2.5-\mathrm{mm}$ silica fiber, under the assumption of having slight chirp, using this as field excitations in the extended FDTD (dashed line) (Nakamura et al., 2005a) and the simpler JGTH-FDTD (short-dashed line) (Nakamura et al., 2005a), and our previously reported experimental result (Karasawa et al, 2000) (solid line).

the $650-750 \mathrm{~nm}$ of the assumed spectrum is zero for the solid line, however, the intensity in the wavelength region is represented by a dashed line, and disagreement could be expected at $650-750 \mathrm{~nm}$ in the calculated results. The inverse Fourier transformation of the assumed input spectrum (dashed line in Fig. 3) indicates a pulse width of $8.5 \mathrm{fs}$ (FWHM), which is the same as that of the experimental spectrum (solid line in Fig. 3). In addition, the output pulse was characterized by our second-harmonic generation frequency-resolved optical gating (SHG-FROG) apparatus set up in place of the spectrometer. Figures obtained by the SHGFROG are shown in section 3.2-A.

\subsection{Numerical results}

\section{A. Comparison between Experimental and Numerical Results}

In our calculation for a fused silica fiber, the parameters in Eq. (10) are set as $b_{1}=0.6961663, b_{2}$ $=0.4079426, b_{3}=0.8974794, \lambda_{1}=0.0684043 \mu \mathrm{m}, \lambda_{2}=0.1162414 \mu \mathrm{m}$, and $\lambda_{3}=9.896161 \mu \mathrm{m}$ (Agrawal, 1995), where $\lambda_{i}=2 \pi c / \omega_{i}$ and $c$ is the velocity of light in vacuum. We used the value of the nonlinear refractive coefficient $n_{2}=2.48 \times 10^{-20} \mathrm{~m}^{2} / \mathrm{W}$ from Ref. (Taylor, 1996), and the third-order susceptibility $\chi^{(3)}$ was found to be $\chi^{(3)}=1.85 \times 10^{-22} \mathrm{~m}^{2} / \mathrm{V}^{2}$ at $800 \mathrm{~nm}$, as given by $\chi^{(3)}$ $=(4 / 3) \varepsilon_{0} c n\left(\omega_{0}\right)^{2} n_{2}$, where $\omega_{0}$ is the center angular frequency of the optical pulse. The parameters $\alpha, \tau_{1}$, and $\tau_{2}$ in Eq. (15) are set to be $\alpha=0.7, \tau_{1}=12.2$ fs and $\tau_{2}=32$ fs (Agrawal, 1995) . A single time step of the finite difference is set as $\Delta t=4.4475215 \times 10^{-17} \mathrm{~s}$ at the wavelength of $800 \mathrm{~nm}$. The time step of $\Delta t$ at $800 \mathrm{~nm}$ is defined as the optical cycle at $800 \mathrm{~nm}$ of $2.6666667 \mathrm{fs}$ divided by 60 . In the extended FDTD calculation, we set all parameters to be the same as in our experiment (Nakamura et al, 2002a), (Karasawa et al, 2000).

We compare the FDTD calculation with the experimental result for the pulse peak power of $175 \mathrm{~kW}$ in order to generate an ultrabroad spectrum which can finally be compressed to 7.1 fs (Nakamura et al, 2002) or even shorter. The total fiber length of $L=2.5 \mathrm{~mm}$ corresponds 

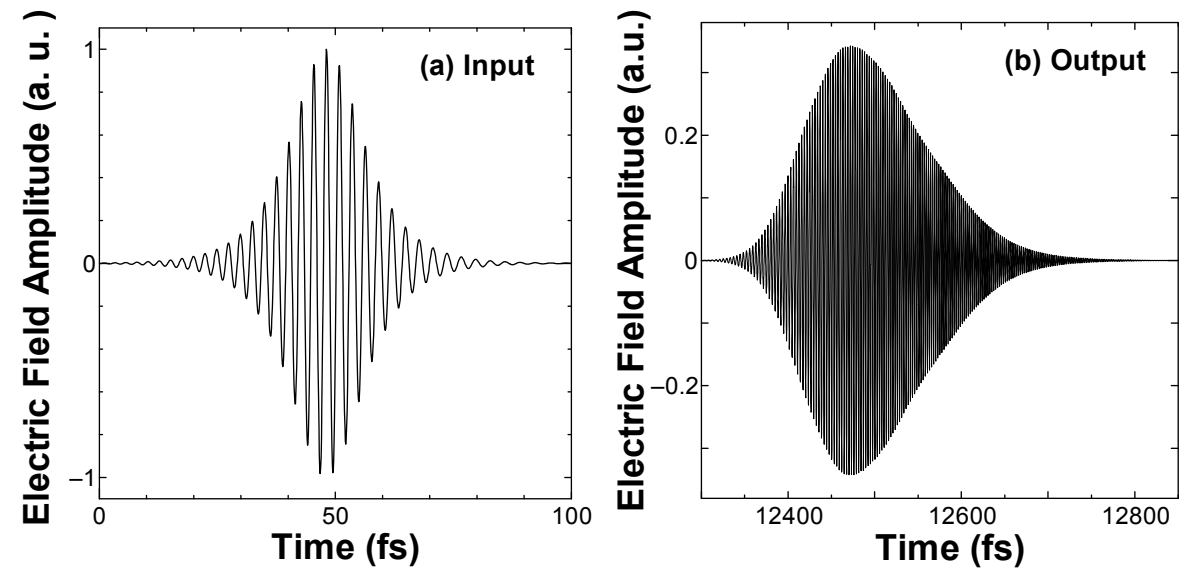

Fig. 4. Input (a) and output (b) temporal electric fields of a $2.5 \mathrm{~mm}$ silica fiber calculated by the extended FDTD Maxwell equation method considering all orders of dispersions and the Raman response [ $\alpha=0.7$ in Eq. (15)]. (Nakamura et al., 2005a)

to 136,500 spatial steps, which means that $L=136,500 \times \Delta z$, where $\Delta z$ is a unit spatial step in the $z$ direction. We need 293,000 time steps to measure the electric field until the pulse tail passes completely. The peak power of an input pulse is set to be $175 \mathrm{~kW}$ (soliton number $N$ = 2.09). The effective core area $A_{\text {eff }}$ is set to be $5.47 \mu \mathrm{m}^{2}$. The iteration number in Eq. (34) is three. An iteration number of two is sufficient, as shown in Refs. (Joseph \& Taflove, 1997) (Goorjian et al., 1992), (Taflove \& Hagness., 2000). Figure 4 shows the input (a) and output (b) temporal electric fields of the fiber. Since the chirp is very small $\left(\partial^{2} \varphi(\omega) /\left.\partial \omega^{2}\right|_{\omega=\omega_{0}}=-\right.$ $\left.113.6 \mathrm{fs}^{2}\right)$, it is seen that the pulse has no chirp in Fig.4(a), while Fig. 4(b) seems to have large chirp because of the linear and nonlinear effects in fiber propagation. Figure 5 shows the temporal intensity profile of the pulse after propagation of the fiber, where the solid line indicates measurement by FROG in the previous experiment, and the dashed line and the short-dashed line indicate the results calculated by the extended FDTD Maxwell equation method and the simpler JGTH-FDTD method, respectively.

The solid line shows a ringing behavior but generally agrees with the calculated result except for the ringing. The envelope of the ringing appears to be the dashed line. The feature of a steep increase (from $-100 \mathrm{fs}$ to $0 \mathrm{fs}$ ) and a slow decrease (from $0 \mathrm{fs}$ to $200 \mathrm{fs}$ ) of the dashed line is also seen in the solid line. The ringing of the experimental result occurs because of the interference between core-mode light and cladding-mode light. Because our simulation is performed by $1 \mathrm{D}$ calculation, the cladding mode is not considered. Hence, there is no ringing in the numerical result. The experimentally measured pulse width (FHWM) is $92 \mathrm{fs}$. Numerically, the pulse width of FWHM is obtained as $108 \mathrm{fs}$. Therefore, the pulse widths are in good agreement with each other. However, the pulse width (FWHM) obtained by the simpler JGTH-FDTD method is $146 \mathrm{fs}$, which is 1.5 times the experimental result. The pulse width obtained by JGTH-FDTD does not agree with the experimental result. This disagreement is caused by the approximation of dispersion in JGTH-FDTD as described in section 3.2. Hence, the extended FDTD is superior to the simpler JGTH-FDTD method. 


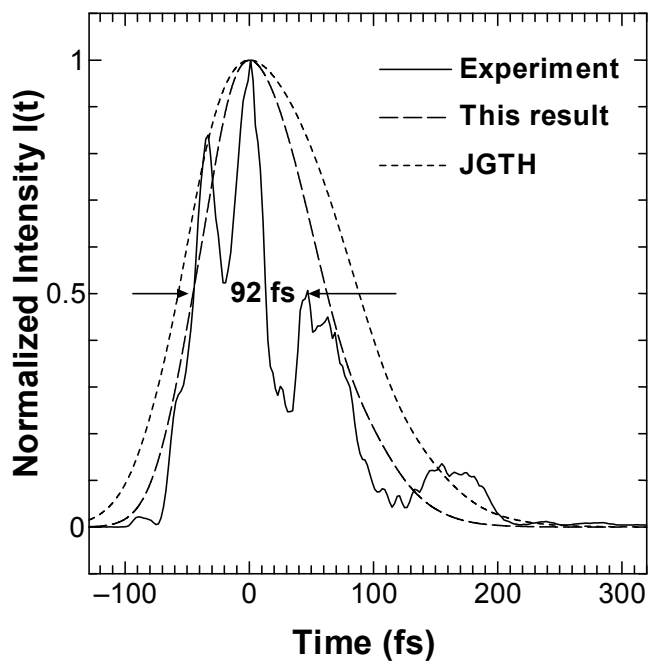

Fig. 5. Temporal intensity profile obtained experimentally (Nakamura et al., 2002) (solid line) and numerically by the extended FDTD (dashed line) and the simpler JGTH-FDTD (short-dashed line) as a function of time for $12 \mathrm{fs}$ laser transmission through a 2.5-mm silica fiber. (Nakamura et al., 2005a)

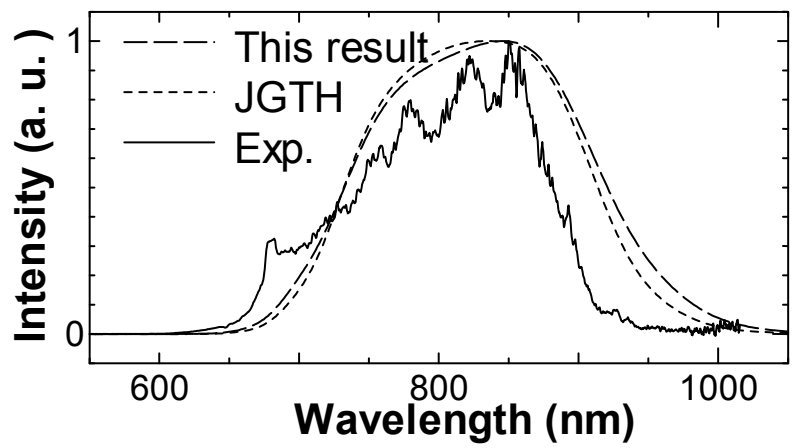

Fig. 6. Spectra after propagation of a slightly chirped $12 \mathrm{fs}$ laser pulse through a 2.5-mm silica fiber, calculated by the extended FDTD (dashed line) (Nakamura et al., 2005a) and the simpler JGTH-FDTD (short-dashed line) (Nakamura et al., 2005a), and our previously reported experimental result (Karasawa et al., 2000) (solid line).

Figure 6 shows the results calculated by the extended FDTD Maxwell equation method (dashed line) and the simpler JGTH-FDTD method (short-dashed line), and our previously reported experimental result (solid line, experiment [Exp.]) (Karasawa et al, 2000). The spectra obtained by the extended FDTD and the simpler JGTH-FDTD show similar results. The trapezium form of the FDTD results is roughly similar to that of the experimental result. Several peaks in the experimental result appear due to interference between core-mode light and cladding-mode light. In BPM solving GNLSE by SSF with SVEA, the second derivative of the electric field with respect to $z, \partial^{2} E_{y} / \partial z^{2}$, is neglected, which corresponds to 
neglecting the backward propagation wave. On the other hand, our extended FDTD Maxwell equation method accurately includes the delayed Raman response and all orders of the dispersion in silica using Sellmeier's equation, and does not use SVEA. The shortest wavelengths (intensity of $1 \%$ ) of the spectra obtained by the extended FDTD, the simpler JGTH-FDTD, and the experimental result (Exp.) are $653 \mathrm{~nm}, 660 \mathrm{~nm}$ and $626 \mathrm{~nm}$, respectively. The longest wavelengths of the extended FDTD, the simpler JGTH-FDTD, and the experimental result (Exp.) are $1046 \mathrm{~nm}, 1027 \mathrm{~nm}$, and $972 \mathrm{~nm}$ (maximum measured wavelength of the spectrometer), respectively. The spectral bandwidths $\Delta \lambda(1 \%$ of peak intensity) of the extended FDTD, the simpler JGTH-FDTD, and the experimental result (Exp.) are $393 \mathrm{~nm}, 367 \mathrm{~nm}$, and $346 \mathrm{~nm}$, respectively. Thus, the spectrum bandwidth of the experimental result (Exp.) is narrower than those of the extended FDTD and the simpler JGTH-FDTD. Note that the intensity at $650-750 \mathrm{~nm}$ of the assumed spectrum is lower than the experimental result, and also that the intensity at $650-750 \mathrm{~nm}$ of the assumed spectrum of the input pulse is zero; however, the intensity of the region of the wavelengths is not zero in the experimental result of the input pulse. This affects the weak intensity at $650-750 \mathrm{~nm}$ in the fiber output spectrum obtained by the extended FDTD and the simpler JGTH-FDTD. In considering wavelengths longer than $800 \mathrm{~nm}$ in Fig. 6, the intensity of the experimental data is lower than those of the extended FDTD and the simpler JGTH-FDTD results. We assume that the sensitivity of the spectrometer detector is low at wavelengths longer than $800 \mathrm{~nm}$ because the detector is made of silicon. We believe our extended FDTD result is correct. We suggest that it is better to use an IR detector made of a material such as InGaAs for the spectrometer.

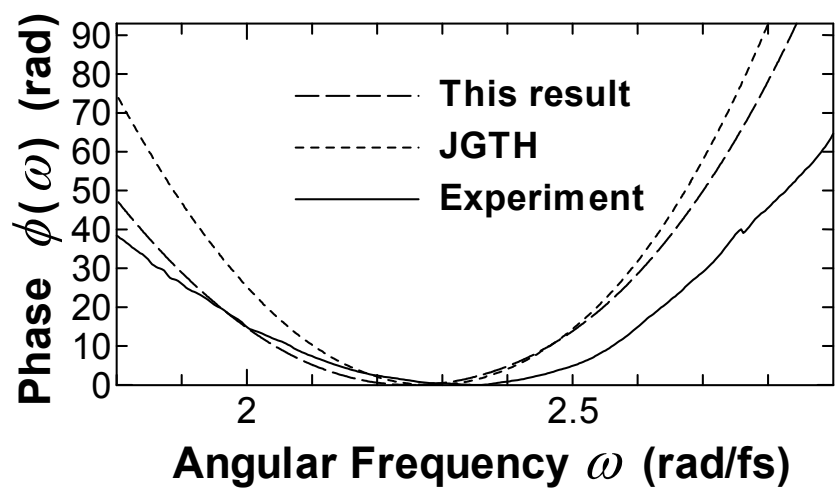

Fig. 7. Spectral phase as a function of angular frequency after a chirped $12 \mathrm{fs}$ pulse propagation through a $2.5-\mathrm{mm}$ silica fiber previously obtained experimentally (solid line)(Nakamura et al., 2002) and numerically by the extended FDTD (dashed line) (Nakamura et al., 2005a) and the simpler JGTH-FDTD (short-dashed line) (Nakamura et al., 2005a)

Figure 7 shows the spectral phase obtained experimentally by the FROG (solid line) and numerically by the extended FDTD (dashed line) and the simpler JGTH-FDTD (shortdashed line) as a function of angular frequency for 12 fs laser transmission through a 2.5mm silica fiber. From our previous experimental result corresponding to the solid line, we 
determine the group-delay dispersion (GDD) and the third-order dispersion (TOD) values to be $342 \mathrm{fs}^{2}$ and $187 \mathrm{fs}^{3}$, respectively. From our calculation corresponding to the dashed line, we determine the GDD and TOD values to be $518 \mathrm{fs}^{2}$ and $333 \mathrm{fs}^{3}$, respectively. The calculated GDD and TOD values do not agree with the experimental results. We have not yet clarified the reason for this disagreement. We must consider assuming the sech ${ }^{2}$ temporal shape of the input pulse, but not the complex waveform obtained by the inverse Fourier transform of the experimentally observed spectrum. However, the calculated result by the extended FDTD (dashed line) from the angular frequency of $1.8 \mathrm{rad} / \mathrm{fs}$ to $2.4 \mathrm{rad} / \mathrm{fs}$ (lower frequency) appears to be in good agreement with the experimental result. On the other hand, the simpler JGTH-FDTD (short-dashed line) shows a large difference in not only the higher angular frequency but also the lower angular frequency. The phase values at the lowest angular frequency of the extended FDTD, the simpler JGTH-FDTD, and the experimental result are $47.4 \mathrm{rad}, 74.2 \mathrm{rad}$, and $38.9 \mathrm{rad}$, respectively. In this comparison, the phase value of the simpler JGTH-FDTD is twice that of the experimental result. This marked difference between the simpler JGTH-FDTD and the experimental result is caused by the dipersion problem of the simpler JGTH-FDTD, which is described in section II and above in this section. The spectral phase is very important for single-cycle pulse generation via phase compensation of the nonlinear-chirped supercontinuum generated in a silica fiber. Hence, the extended FDTD is superior to the simpler JGTH-FDTD in predicting the spectral phase for the phase compensation.

\section{B. Compensation of group delay by using SLM}

We assume the model in which the output beam from the fiber is collimated by a spherical mirror and is directed to a $4-f$ system by the SLM. The 4- $f$ system consisted of two spherical mirrors and two reflective gratings (Nakamura et al, 2002a), (Karasawa et al, 2001), (Yamane et al., 2003). The optical path lengths from the gratings to the spherical mirrors with a focal length $f$ and those from the spherical mirrors to the SLM are all set to be $f$. The liquid crystal SLM (Citizen Company) (Karasawa et al, 2001) consisted of $64897-\mu \mathrm{m}$-wide pixels, with a 5 $\mu \mathrm{m}$ gap between adjacent pixels.

The SLM is used as a phase modulator, which can impose a phase variation of $2 \pi$ on light whose wavelength is less than $1400 \mathrm{~nm}$ with a resolution of 192 (Karasawa et al, 2001). The phase $\phi_{\mathrm{SLM}}(x)$ from the SLM at position $x$ is given by a polynomial of the form

$$
\phi_{\mathrm{SLM}}(x)=\frac{\alpha}{2}\left[\omega(x)-\omega_{0}\right]^{2}+\frac{\beta}{6}\left[\omega(x)-\omega_{0}\right]^{3}+\frac{\gamma}{24}\left[\omega(x)-\omega_{0}\right]^{4},
$$

where $\omega_{0}$ is the center angular frequency for a Taylor expansion, $\omega(x)$ is the angular frequency at position $x$ and is given by $\omega(x)=2 \pi c /\left\{d \sin \left[\tan ^{-1}(x / f)+\sin ^{-1}\left(\lambda_{c} / d\right)\right]\right\}, c$ is the speed of light, and $\lambda_{c}=800 \mathrm{~nm}$ is the wavelength at the central position of the SLM, where $x$ $=0$. $\lambda_{c}$ does not have to be equal to the center wavelength for the Taylor expansion $\lambda_{0}$, where $\lambda_{0}=2 \pi c / \omega_{0} . \alpha, \beta$, and $\gamma$ are GDD, TOD, and the fourth-order dispersion (FOD), respectively, at $\omega_{0}$. The group delay $t_{\mathrm{d}, \mathrm{SLM}}(x)$ imposed by the SLM is

$\mathrm{t}_{\mathrm{d}, \mathrm{SLM}}(x)=\frac{\partial \varphi_{\text {SLM }}(\omega)}{\partial \omega}$

$$
=\alpha\left[\omega(x)-\omega_{0}\right]+\frac{\beta}{2}\left[\omega(x)-\omega_{0}\right]^{2}+\frac{\gamma}{6}\left[\omega(x)-\omega_{0}\right]^{3} .
$$


Parameters $\alpha, \beta$, and $\gamma$ were initially estimated from the total group delay $t_{d}(\omega)$ of a fiber (including the self-phase modulation). $t_{d}(\omega)$ is fitted to the form of Eq. (36), and $\alpha, \beta$, and $\gamma$ are obtained. Then the negative values of these fitted parameters were initially imposed by the SLM to satisfy $t_{d, \mathrm{SLM}}(\omega)+t_{d}(\omega)=$ constant (zero in our case) in the entire frequency range. Theoretically, this condition should produce the shortest pulses. Use of the Taylor expansion in Eq. (35) is found to be the most suitable way to perform fine adjustment of the phase. Parameters $\alpha, \beta$, and $\gamma$ are fine-tuned by a computer to yield the shortest pulse, with the constant group delay dispersion optimized as a function of wavelength. Figure 8 shows the calculated group delay after the pulse propagation of a fiber (solid curves) and the compensated group delay (dashed curve) with the negative value of the optimized group delay applied by the SLM. The group delay after fiber propagation is added by applying its negative value to the SLM, and then the total of these values is shown as a dashed line for which the shortest pulse (a nearly transform-limited pulse) is obtained for the condition where $\lambda_{0}$ is set to $800 \mathrm{~nm}$. The temporal intensity profile of this shortest pulse is shown in Fig. 9. The pulse width (FWHM) is estimated to be $6.9 \mathrm{fs}$. This pulse width agrees with the compressed pulse of $7.1 \mathrm{fs}$ (Nakamura et al, 2002a) previously obtained experimentally by using a combination of prism compression and a 256 pixel SLM. This finding indicates that the combination of this extended FDTD and group delay compensation simulation is robust for estimating the shortest pulse characteristics after the propagation of a fiber, and useful for constructing a fiber-compression system for obtaining a single-cycle pulse.

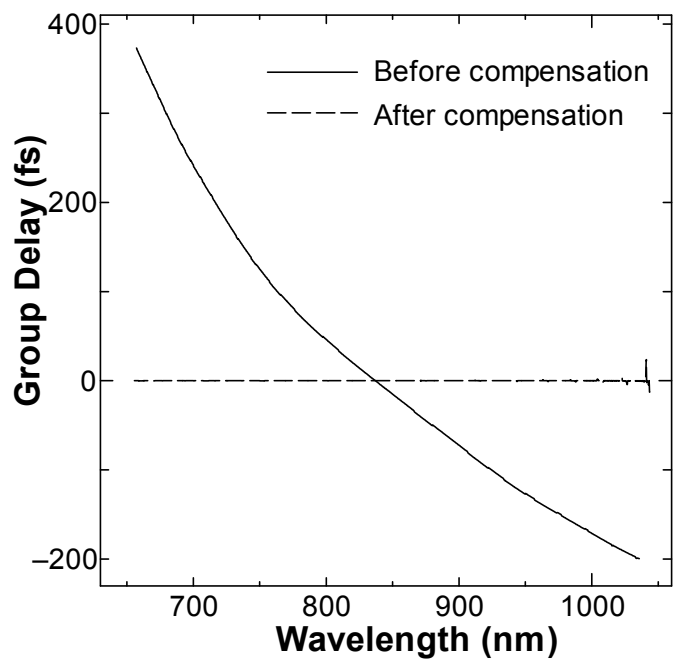

Fig. 8. Calculated group delay after pulse propagation of a fiber (solid curves) and the compensated group delay (dashed curve) with the negative of the optimized group delay applied by the SLM. (Nakamura et al., 2005a) 


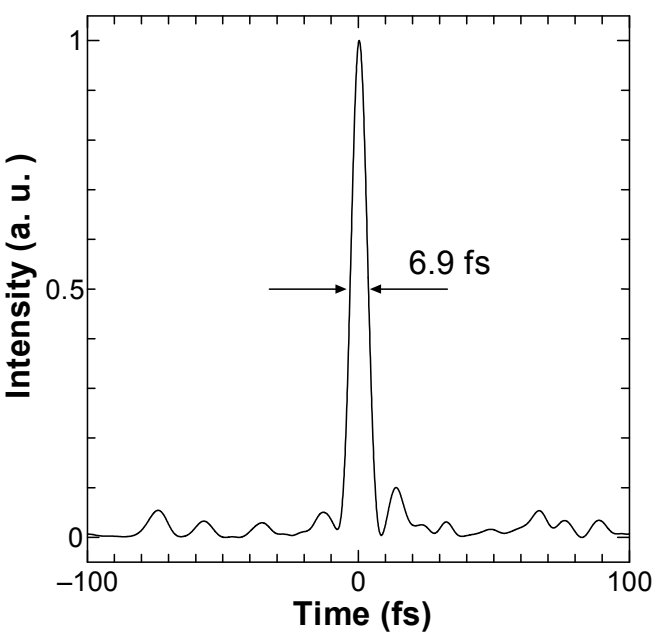

Fig. 9. Temporal time profile compensated optimally by SLM after propagation of a fiber. The pulse width (FWHM) is 6.9 fs. (Nakamura et al., 2005a)

\subsection{Summary of single pulse propagation}

We have extended the nonlinear FDTD method with a JGTH algorithm to one with exact Sellmeier's fitting values in order to compare the experimental and calculated results of nonlinear femtosecond ultrabroadband-pulse propagation in a silica fiber, and have analyzed the temporal and spectral characteristics of the propagated pulse. This extended method is robust against the breakdown of the SVEA. The spectrum, temporal intensity profile, and pulse width after phase compensation results as calculated by the extended FDTD method agree well with those obtained in our previous experiment. These findings indicate that the combination of this extended FDTD method and the group delay compensation simulation is robust for estimating the spectrum, the temporal pulse profile after propagation through a fiber and the shortest pulse obtained by compensation, and is useful for estimating the shortest pulse when the fiber-compression system is designed to obtain a single-cycle pulse (Yamane et al., 2003).

When the extended FDTD method is established as the ultrashort optical pulse propagation analysis technique, its application to the characteristic analysis of an ultrahigh-speed optical switch, the propagation characteristic analysis of the optical pulse in a photonic crystal, and nonlinear propagation analysis in a photonic crystal fiber or a taper fiber are expected.

\section{Dual wavelengths femtosecond pulses propagation}

\subsection{Induced-phase modulation}

Recently, there has been significant interest in the generation of single-cycle optical pulses by optical pulse compression of ultrabroadband light produced in fibers. The spectrabroadening by the induced-phase modulation (IPM) is one of these methods. There have been some reports of experiments on ultrabroadband-pulse generation using IPM in a silica fiber (Xu et al., 1999), (Karasawa et al., 2001b), (Shibata et al., 2002), (Yamashita et al., 2004) and an Ar-gas-filled hollow fiber, (Karasawa et al., 2000b) and also on optical pulse 
compression by nonlinear chirp compensation. (Yamashita et al., 2004) For these experiments on generating few-optical-cycle pulses, characterizing the spectral phase of ultrabroadband pulses analytically as well as experimentally is very important.

In IPM calculation, details of the phase characteristics at the connecting point of the two pulses are not clearly understood because the beam propagation method (BPM) has been used to describe the propagation of an optical pulse in a fiber. (Agrawal, 1995) In the BPM, each phase curve is obtained independently as a function of frequency for each center frequency of the two pulses. (Agrawal, 1995) The two phase curves are connected at the same phase value, as reported in refs. (Yamashita et al., 1998) and (Yamashita et al., 1996). Recently, we extended (Nakamura et al., 2002b) the finite-difference time domain (FDTD) method with nonlinear polarization $P_{N L}$ involving the Raman response function to $12 \mathrm{fs}$ ultrabroadband-pulse propagation in a silica fiber with the consideration of linear polarization $P_{L}$, including all exact Sellmeier-fitting values of silica with three resonant frequencies, in order to compare the calculated results with our experimental results. (Nakamura et al., 2002a), (Karasawa et al., 2000) This extended FDTD method (Nakamura et al., 2002b) is more accurate than previous FDTD methods (Joseph \& Taflove, 1997), (Kalosha \& Herrmann), (Goorjian et al., 1992), (Joseph et al., 1993), (Taflove \& Hagness, 2000). Recently, a similarly accurate algorithm, which assumes that the linear and nonlinear dispersions are modeled by three and one Lorentzian resonances, respectively, was suggested by Goojian and Cundiff (Goojian \& Cundiff, 2004). On the point of phase observation, the extended FDTD is superior to BPM (Yamashita et al., 1998) and (Yamashita et al., 1996) because the extended FDTD method includes no assumption for the two-pulse case, which implies that it can be used to calculate two different pulses simultaneously. We have recently numerically obtained the phase when two different pulses were simultaneously injected into a fiber and IPM occurred, and we clarified how the two pulse phases maintain their connection with each other (Nakamura et al., 2004a). We did not observe the four-wave mixing but only SPM and IPM with the simultaneous injection of two-color pulses into a fiber (Nakamura et al., 2004a). In this study, we observed the anti-Stokes light of the degenerate four-wave mixing (DFWM) in the IPM spectral phase obtained by the extended FDTD method with an initial delay of $-80 \mathrm{fs}$. The chirped first and second pulses broadening with dispersion interact with each other, and the nonlinear interaction length is prolonged with this initial delay. Then the phase matching condition for DFWM at the end of the fiber is satisfied with an initial delay of $-80 \mathrm{fs}$, which corresponds to the situation of two pulses meeting at the end of the fiber via a different group velocity of the two pulses. This is the first observation of the DFWM with IPM by FDTD. Furthermore, we compared the extended FDTD method (Nakamura et al., 2002b), (Nakamura et al., 2004b), (Nakamura et al., 2005) with experimental results (Xu et al., 1999) for nonlinear propagation with the IPM effect of two-color sub-picosecond laser pulses in a silica fiber that have different initial delays between the two pulses. To the best of our knowledge, this is the first comparison between FDTD calculation and experimental results for nonlinear propagation with the IPM effect of twocolor 120 fs laser pulses in a silica fiber. The extended FDTD method clarifies how the two pulse phases maintain their connection with each other with three initial time delays.

\subsection{Experimental setup}

A schematic of our experimental setup (Xu et al., 1999) is shown in Fig. 10. The first pulse was generated from a Ti:sapphire regenerative amplifier at a $1 \mathrm{kHz}$ repetition rate. 


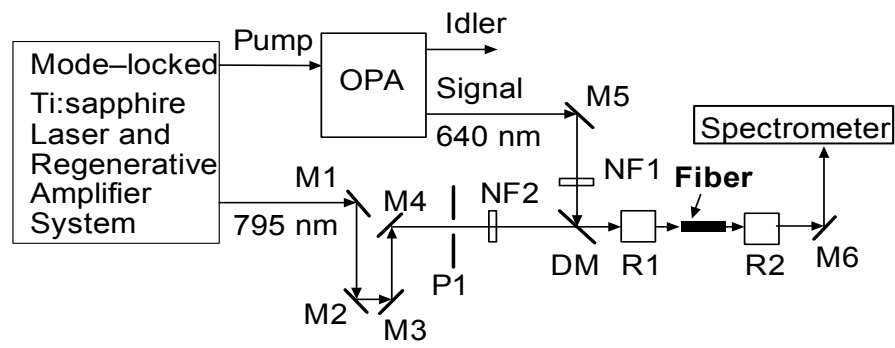

Fig. 10. Layout of the experimental setup.(Xu et al., 1999) M, silver-coated plane mirrors; R1 and R2, reflective objectives (36x); DM, dichroic mirror; NF1 and NF2, valuable optical attenuators; OPA, optical parametric amplifier.

The pulse duration was about $120 \mathrm{fs}$ full width at half maximum (FWHM) with a bandwidth of $12 \mathrm{~nm}$ FWHM centered at $795 \mathrm{~nm}$, and the output energy was $750 \mu \mathrm{J}$, henceforth called the fundamental pulse. The main output energy from the regenerative amplifier was used to generate a continuum wave and pump an optical parametric amplifier (OPA) for the second pulse generation. The tuning range in the OPA was from 480 to $740 \mathrm{~nm}$ with an output energy of over $10 \mu \mathrm{J}$ at $1 \mathrm{kHz}$. In this experiment, we set the wavelength of the second pulse at $640 \mathrm{~nm}$ with a $120 \mathrm{fs}$ FWHM in the pulse duration and a $12 \mathrm{~nm}$ FWHM in the spectral width, henceforth called the signal pulse. Since the continuum wave was generated by the SPM of the fundamental pulse under the single-filament condition and was selectively amplified using its second harmonic pulse by the optical parametric process with the phase matching condition, the phase of the signal wave should have a relationship to the phase of the fundamental wave. This condition is critical for generating a stable combined spectrum with IPM in a single-mode glass fiber, which will be confirmed later. The calculated walk-off length (Agrawal, 1995) $L_{w}$ between the signal pulse and the fundamental pulse was about $2.7 \mathrm{~mm}$. The reason the second pulse wavelength was selected to be $640 \mathrm{~nm}$ lies in the fact that the walk-off length $L_{w}$ of these two pulses in a fused silica fiber $\left(L_{w}=2.7 \mathrm{~mm}\right)$ is around the length that we are able to cut easily, about $3 \mathrm{~mm}$, and the IPM effect between the two pulses can be observed clearly at this length. The two pulses were combined by a dichroic mirror, DM, which has a high transmission for the fundamental pulse and a high reflection for the signal pulse. The optical path of the fundamental pulse was varied by an optical delay stage formed by M2 and M3 so that the delay of the two pulses in the fiber could be adjusted. N1 and N2 are variable neutral-density filters and the energies of the two pulses can be controlled independently. The combined two pulses, which have the same linear polarizations, were coupled into a $3 \mathrm{~mm}$ single-mode polarization-preserving fusedsilica fiber $(2.7 \mu \mathrm{m}$ core diameter, $550 \pm 50 \mathrm{~nm}$ cutoff wavelength) by a $36 \times$ reflective objective, R1. The output from the fiber was collimated by the same type of reflective objective, R2, to a spectrometer that monitored the output spectrum.

\subsection{Numerical results}

In our calculation for a fused silica fiber, the parameters in eq. (10) are set as $b_{1}=0.6961663$, $b_{2}=0.4079426, b_{3}=0.8974794, \lambda_{1}=0.0684043 \mu \mathrm{m}, \lambda_{2}=0.1162414 \mu \mathrm{m}$, and $\lambda_{3}=9.896161 \mu \mathrm{m}$ (Agrawal, 1995), where $\lambda_{i}=2 \pi c / \omega_{i}$ and $c$ is the velocity of light in vacuum. We used the 
value of the nonlinear refractive coefficient $n_{2}^{I}=2.48 \times 10^{-20} \mathrm{~m}^{2} / \mathrm{W}$ from ref. (Taylor et al., 1996), and the third-order susceptibility $\chi^{(3)}$ was found to be $\chi^{(3)}=1.85 \times 10^{-22} \mathrm{~m}^{2} / \mathrm{V}^{2}$ at 800 $\mathrm{nm}$, given by $\chi^{(3)}=(4 / 3) \varepsilon_{0} c n\left(\omega_{0}\right)^{2} n_{2}^{I}$, where $\omega_{0}$ is the center angular frequency of the optical pulse. The parameters $\alpha, \tau_{1}$, and $\tau_{2}$ in eq. (15) are set to be $\alpha=0.7, \tau_{1}=12.2 \mathrm{fs}$ and $\tau_{2}=32 \mathrm{fs}$. (Agrawal, 1995) A single time step of the finite difference is set as $\Delta t=7.116034310 \times 10^{-17} \mathrm{~s}$. The time step of $\Delta t$ is defined as the single optical cycle divided by 60 . In the extended FDTD calculation for two-pulse propagation in a silica fiber, we set all other parameters to be the same as those in the previous IPM experiment. (Xu et al., 1999) Both pulses had the same pulse width of $120 \mathrm{fs}$ and the same energy of $20 \mathrm{~nJ}$. The fiber was 3-mm long with a core diameter of $2.7 \mu \mathrm{m}$. The fiber length of $3 \mathrm{~mm}$ nearly equals the walk-off length of 2.7 $\mathrm{mm}$. The effective core area $A_{\text {eff }}$ was $5.47 \mu \mathrm{m}^{2}$. Two laser pulses at the center wavelengths of 795 and $640 \mathrm{~nm}$ (the fundamental and signal pulse) propagated in a $3 \mathrm{~mm}$ silica fiber with the delay of the signal pulse for the fundamental pulse, $T_{d}=0,-40,-80 \mathrm{fs}$. The delay of $-40 \mathrm{fs}$ corresponds to the situation in which the two pulses are launched simultaneously into the same fiber with an optimum initial delay between the two pulses that meet at the center of the fiber, to be discussed later, because of the IPM effect of the two pulses, the two spectra meet each other. The delay of $-80 \mathrm{fs}$ corresponds to the situation which two pulses meet at the end of the fiber. Since every delay case is performed close to the walk-off length, the nonlinear interaction length is sufficient.
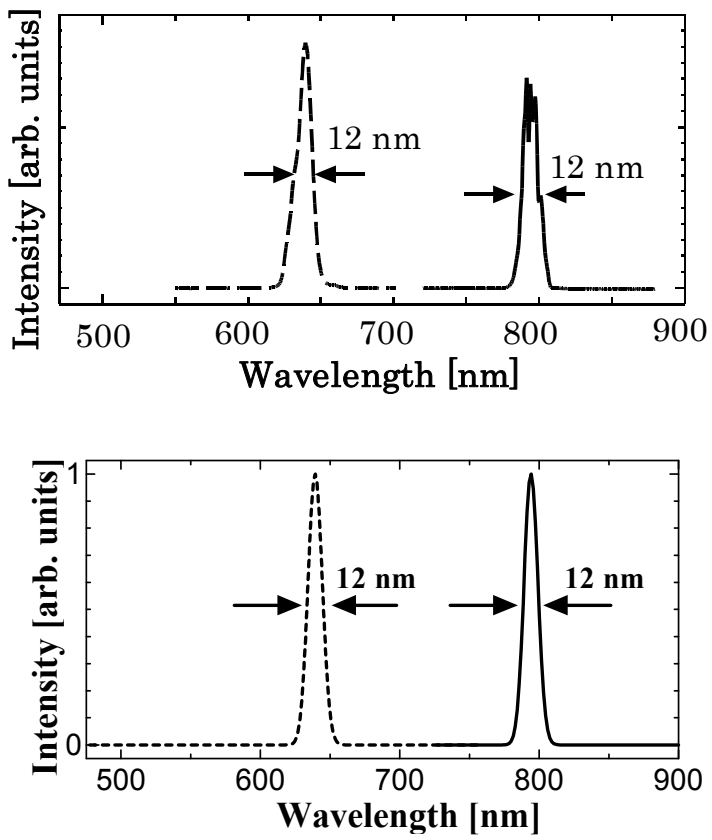

Fig. 11. Spectra obtained (a) experimentally (Xu et al., 1999) and (b) numerically (Nakamura et al., 2005b) for two pulses at center wavelengths of $795 \mathrm{~nm}$ (solid line) and $640 \mathrm{~nm}$ (dashed line) before propagation in a $3 \mathrm{~mm}$ silica fiber. 
Figure 11 shows (a) the experimentally measured and (b) the numerically assumed spectra of the input fundamental (solid line) and signal (dashed line) pulses. The FWHM spectral width of $\Delta \lambda$ is $12 \mathrm{~nm}$ for both fundamental and signal pulses. The Fourier transform of a Gaussian spectrum with the FWHM spectral width of $\Delta \lambda=12 \mathrm{~nm}$ for the fundamental and signal pulses leads to a pulse width of 80.0 and $50.0 \mathrm{fs,} \mathrm{respectively,} \mathrm{which} \mathrm{does} \mathrm{not} \mathrm{agree}$ with the input pulse width of $120 \mathrm{fs}$. This fact indicates that the input pulses are chirped, which accords with the second derivation of the spectral phase of $\partial^{2} \varphi(\omega) /\left.\partial \omega^{2}\right|_{\omega=\omega_{0}}=4651$ and $2382 \mathrm{fs}^{2}$ for the fundamental and signal pulses, respectively. Fig. 11(b) is assumed by the Fourier transformation of the pulse width of $120 \mathrm{fs}$ with the chirp values. Due to the group velocity difference between the two optical pulses propagating in the fiber, they travel at different speeds and after some propagation distance they are separated. We measured the IPM-induced spectra with different initial time delays between the two pulses.

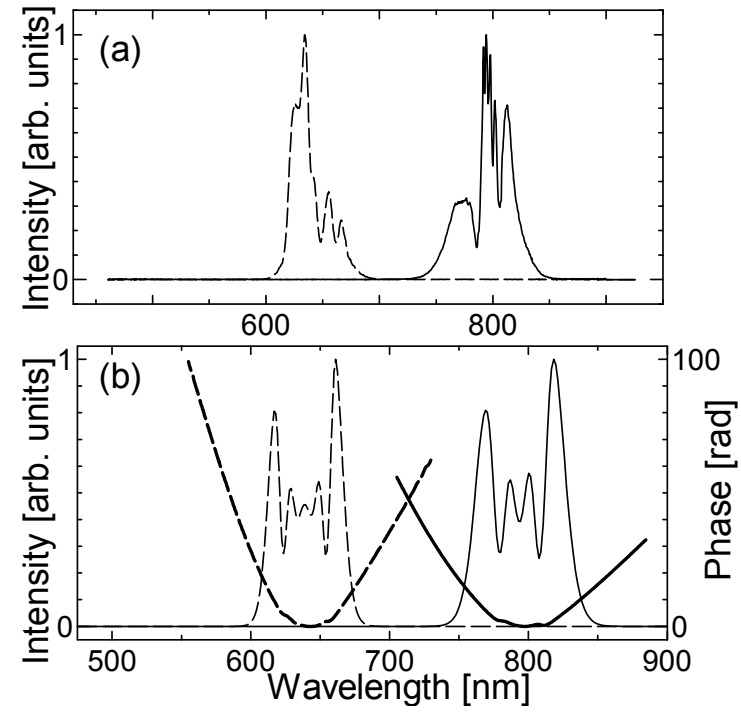

Fig. 12. (a) Experimentally (Xu et al., 1999) and (b) numerically (Nakamura et al., 2005b) obtained spectra induced by SPM in $3 \mathrm{~mm}$ single-mode fused-silica fiber. The thin solid line represents the spectrum induced by the SPM of the pulse at the center wavelength of $795 \mathrm{~nm}$ and the thin dashed line represents the spectrum induced by the SPM of the pulse at the center wavelength of $640 \mathrm{~nm}$ at the pulse duration of $120 \mathrm{fs}$ and the energy of $20 \mathrm{~nJ}$ in the 3 $\mathrm{mm}$ fiber for each pulse. The thick lines in (b) are spectral phases obtained numerically as a function of wavelength for the $3 \mathrm{~mm}$ fiber-propagated pulses at center wavelengths of 795 $\mathrm{nm}$ (thick solid line) and $640 \mathrm{~nm}$ (thick dashed line).

Fig. 12 depicts the spectra of the two pulses with SPM alone at the energy of $20 \mathrm{~nJ}$ in the fiber for each pulse, which are obtained (a) experimentally and (b) numerically. The solid line represents the SPM-induced spectrum of the fundamental pulse and the dashed line represents the SPM-induced spectrum of the signal pulse. From Figs. 12(a) and 12(b) we observe the separated spectra for the two pulses with only the SPM effect at $20 \mathrm{~nJ}$ energy levels. Here, we define the wavelengths in which the spectral intensity becomes 1/100 of the 
maximum intensity as $\lambda_{\min }$ and $\lambda_{\max }$ for the shortest and longest wavelength, respectively. Then, we define the spectral width as $\Delta \lambda_{1 / 100}=\lambda_{\max }-\lambda_{\min }$. The spectral widths $\Delta \lambda_{1 / 100}$ of the dashed lines in Figs. 12(a) and 12(b) are 93.8 and $83.7 \mathrm{~nm}$, respectively. The spectral widths $\Delta \lambda_{1 / 100}$ of the solid lines in Figs. 12(a) and 12(b) are 117.5 and $110.9 \mathrm{~nm}$, respectively. The spectral widths in Fig. 12 caused by only SPM are smaller than those caused by SPM and IPM of Figs. 13 and 14, this will be discussed later. The difference in the spectral width between the experimental and numerical results is less than $10 \mathrm{~nm}(<10 \%)$, which indicates that the difference is small. Blue shift due to self-steepening is not observed but breakdown of the spectral symmetry is observed. The intensity of the spectrum is higher for wavelengths longer than the center wavelengths in Fig. 12(b). In particular, the solid line (fundamental pulse) in Fig. 12(b) shows asymmetrical behavior and three- or four-peak behavior, which are similar to those in the solid line in Fig. 12(a). The extended FDTD result and the experimental result in Fig. 12 are similar from the point of view of spectral widths. The thick lines in Fig. 12(b) show the numerically obtained spectral phase wherein the dashed line shows the phase of the fundamental pulse and the solid line shows that of the signal pulse. For both the fundamental and signal pulse, the phase curve is a symmetric parabolic curve. The spectral phases of the two pulses cross around the wavelength of 713 $\mathrm{nm}$, where the phase value is $48.0 \mathrm{rad}$.

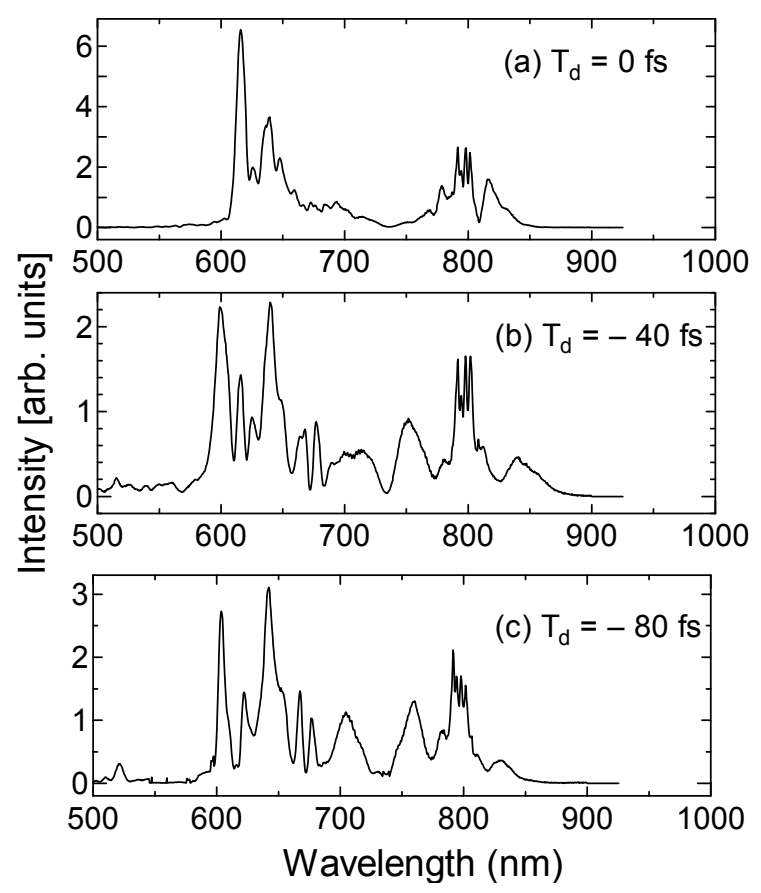

Fig. 13. Experimentally measured spectra (Xu et al., 1999) induced by IPM for two pulses at center wavelengths of 795 and $640 \mathrm{~nm}$ with different initial time delays after propagation in $3 \mathrm{~mm}$ silica fiber. (a) Spectrum when two pulses meet at entrance of fiber, (b) spectrum when two pulses meet at center of fiber and (c) spectrum when two pulses meet at end of fiber. 


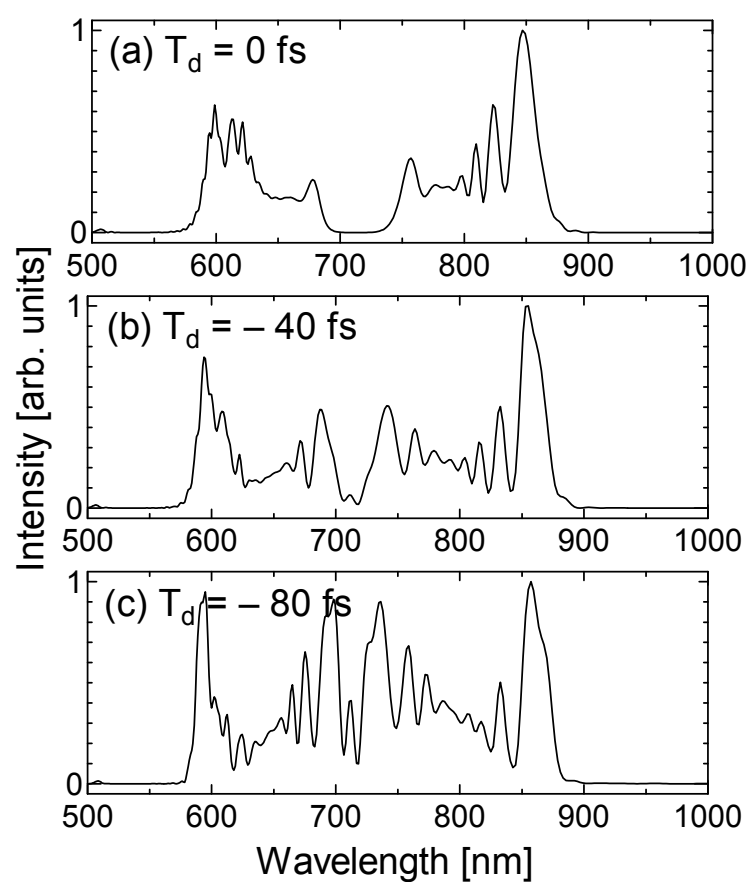

Fig. 14. Numerically obtained spectra induced by IPM for two pulses at center wavelengths of 795 and $640 \mathrm{~nm}$ with different initial time delays after propagation in $3 \mathrm{~mm}$ silica fiber. (a) Spectrum when two pulses meet at entrance of fiber, (b) spectrum when two pulses meet at center of fiber and (c) spectrum when two pulses meet at end of fiber. (Nakamura et al., 2005b)

Figures 13 and 14 show the IPM-induced spectra obtained by the previous experiment $(\mathrm{Xu}$ et al., 1999) and the extended FDTD calculation with different initial time delays between the two pulses, these figures indicate spectra after propagation of $3 \mathrm{~mm}$ fiber with the initial delays $T_{d}$ of (a) 0 , (b) -40 , and (c) $-80 \mathrm{fs}$, respectively. The pulse energies after fiber propagation of the two pulses are the same value, $40 \mathrm{~nJ}$. The initial time delay between the two pulses, (a) $T_{d}=0 \mathrm{fs}$, represents that the two pulses coincide at the fiber entrance, and the delays, (b) $T_{d}=-40 \mathrm{fs}$ and (c) $T_{d}=-80 \mathrm{fs}$, represent that the two pulses meet at the center and the end of the fiber, respectively. Figures 13 and 14 indicate that the spectra are all broader than that of Fig. 12, because not only SPM but also IPM occurs. The spectral bandwidth of Fig. 13(a) is $\Delta \lambda_{1 / 100}=\lambda_{\max }-\lambda_{\min }=851.1-568.9=282.2 \mathrm{~nm}$. The spectral bandwidth of Fig. 14(a) is $\Delta \lambda_{1 / 100}=\lambda_{\max }-\lambda_{\min }=883.8-571.5=312.3 \mathrm{~nm}$. Quantitatively, there is a difference between the experiment [Fig. 13(a)] and the calculation [Fig. 14(a)]. However, a larger gap between the two spectra is observed in both cases. This shows that the calculation qualitatively agrees well with the previous experimental results. In our case, when the two pulses meet at the entrance of the fiber $T_{d}=0 \mathrm{fs}$, the leading edge of the signal pulse interacts with the trailing edge of the fundamental pulse because the fundamental pulse travels faster than the signal pulse in the fused silica fiber. As a result, both the signal pulse and the fundamental pulse have IPM-induced chirp. This leads to a larger modulation of the signal pulse spectrum at shorter wavelengths and a larger spectral modulation of the fundamental pulse 
at longer wavelengths. Hence, the modulation of the signal pulse at longer wavelengths and of the fundamental pulse at shorter wavelengths is depressed. Then, the larger gap between the fundamental and signal spectra is formed. In contrast, when the two pulses meet at the end of the fiber $T_{d}=-80 \mathrm{fs}$, the trailing edge (longer wavelengths) of the signal pulse mainly interacts with the leading edge (shorter wavelengths) of the fundamental pulse. As a result, the IPM-induced spectrum of the signal pulse shifts towards its longer wavelengths and the IPM-induced fundamental pulse spectrum shifts towards its shorter wavelengths and a larger overlap of the two pulse spectra is observed in both Figs. 13(c) and 14(c). This shows that the calculation qualitatively agrees well with the previous experimental results. The spectral bandwidth of Fig. 13 (c) is $\Delta \lambda_{1 / 100}=\lambda_{\max }-\lambda_{\min }=861.8-582.9=278.9 \mathrm{~nm}$. The spectral bandwidth of Fig. 14 (c) is $\Delta \lambda_{1 / 100}=\lambda_{\max }-\lambda_{\min }=895.9-579.1=316.8 \mathrm{~nm}$. It is found that when the two pulses meet at the center of the fiber, the fundamental pulse passes through the signal pulse in a symmetric manner. As a result, the spectra are broadened by the IPM effect to shorter and longer wavelengths simultaneously for both pulses and the broadest combined spectrum is generated in both Figs. 13(b) and 14(b). This shows that the calculation qualitatively agrees well with the previous experimental results. The spectral bandwidth of Fig. 13(b) is $\Delta \lambda_{1 / 100}=\lambda_{\max }-\lambda_{\min }=887.3-567.3=320.0 \mathrm{~nm}$. The spectral bandwidth of Fig. $14(\mathrm{~b})$ is $\Delta \lambda_{1 / 100}=\lambda_{\max }-\lambda_{\min }=896.6-577.6=319.0 \mathrm{~nm}$. Figures $13(\mathrm{~b})$ and $14(\mathrm{~b})$ generally have the same value of $\Delta \lambda_{1 / 100}$. This case is referred to as the optimum initial time delay to produce the broadest spectrum. In Figs. 13 and 14, it is the same feature that the most broadened spectra are Fig. 13(b) and Fig. 14(b) with an initial delay of $T_{d}=-40$ fs. Consequently, the extended FDTD results and our previous experimental results have the same behavior of spectral bandwidth, which indicates the same initial delay dependence. That is, the extended FDTD calculation qualitatively agrees well with the previous experimental results although it does not agree quantitatively. This quantitative disagreement might be due to the low sensitivity of the detector of the spectrometer at wavelengths longer than $800 \mathrm{~nm}$ because the detector is made of Si. We suggest that InGeAs should be used for the detector, which is highly sensitive in the detection of the infrared region light.

Figure 15 shows the spectral phase obtained numerically as a function of wavelength for mixed two pulses at center wavelengths of 795 and $640 \mathrm{~nm}$ after simultaneous copropagation with an initial delay of (a) 0 , (b) -40 , and (c) $-80 \mathrm{fs}$ in a $3 \mathrm{~mm}$ silica fiber. Around the center wavelength $(795 \mathrm{~nm})$ of the fundamental pulse, the phase curve is parabolic and its symmetry axis is (a) 774.14, (b) 749.45, and (c) $775.58 \mathrm{~nm}$. Around the center wavelength $(640 \mathrm{~nm})$ of the signal pulse, the phase curve is parabolic and its symmetry axis is (a) 673.99, (b) 676.90, and (c) $687.78 \mathrm{~nm}$. The symmetry axis of every parabolic curve in Fig. 15 shifts toward the wavelength of $720 \mathrm{~nm}$, which is the center wavelength between 795 and $640 \mathrm{~nm}$. This feature is seen when the IPM effect occurs, so that this feature is not found only in the SPM case of Fig. 12. In addition, it is observed in Fig. 15 that the spectral phases of the two pulses at the wavelength $\lambda$ of (a) 711.7, (b) 713.17, and (c) $713.17 \mathrm{~nm}$ are continuously connected at a phase value of $\phi(\lambda)=(\mathrm{a}) 17.76$, (b) 10.46, and (c) $17.94 \mathrm{rad}$ without any discrete point. Furthermore, regarding wavelengths shorter than $640 \mathrm{~nm}$, one more parabolic curve is found between 500 and $570 \mathrm{~nm}$. This curve is found to be that of the anti-Stokes light (theoretically $535.58 \mathrm{~nm}$ ) generated by the induced DFWM where the pump light is $640 \mathrm{~nm}$ and the Stokes light is $795 \mathrm{~nm}$. The chirped first and second pulses broadening by dispersion interact each other, and the nonlinear interaction length is prolonged with an initial delay of -80 fs. The DFWM is robust for chirping. (Cundiff et al., 1999), (Geraghty et al., 1998) 

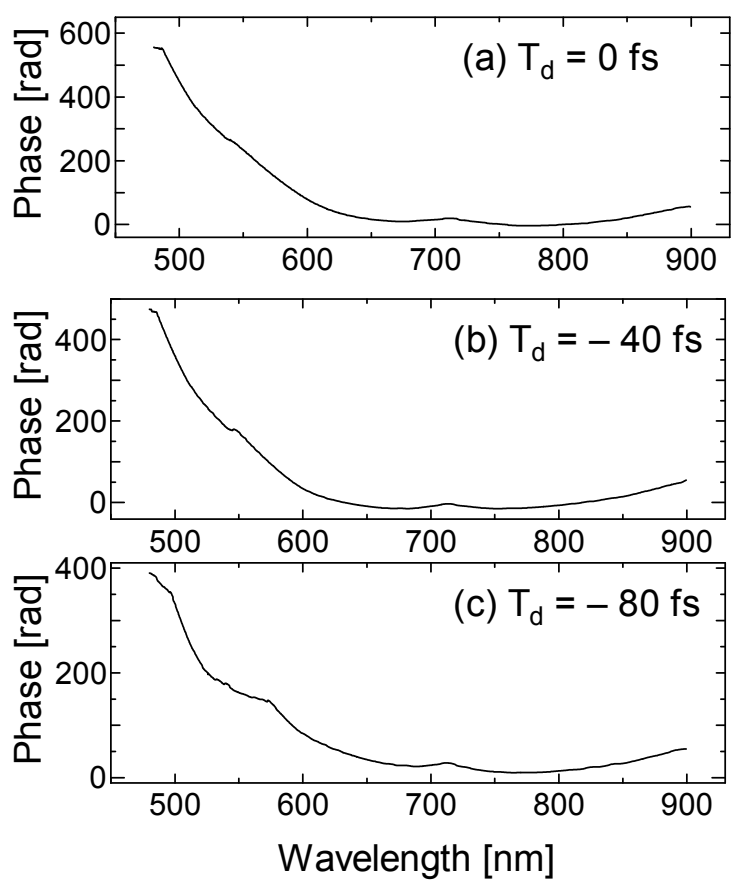

Fig. 15. Spectral phase obtained numerically as function of wavelength for mixed two pulses at center wavelengths of 795 and $640 \mathrm{~nm}$ after simultaneous copropagation with an initial delay of (a) 0 , (b) -40 , and (c) $-80 \mathrm{fs}$ in $3 \mathrm{~mm}$ silica fiber. (Nakamura et al., 2005b)

When the two pulses meet at the end of the fiber $T_{d}=80 \mathrm{fs}$, the trailing edge of the signal pulse mainly interacts with the leading edge of the fundamental pulse and the chirped pulses gradually become overlapping at the places where the pulses are temporally broadened; therefore, the interaction length is long. As a result, the four-wave mixing effect is enhanced and a larger parabolic phase curve of the anti-Stokes light in the spectral phase is observed. Then the phase matching condition for DFWM at the end of the fiber is satisfied with an initial delay of $-80 \mathrm{fs}$, which corresponds to the situation in which two pulses meet at the end of the fiber via a different group velocity of the two pulses. Looking again at Fig. 15 , the anti-Stokes light appears faintly in the phase with an initial delay of -40 as well, but no anti-Stokes light appears with an initial delay of $0 \mathrm{fs}$. The anti-Stokes light of DFWM appears when long chirped pulses finally overlap at the end of the fiber, the IPM effect is strong when short pulses overlap at the beginning of the fiber, and IPM and DFWM crossinteract and spectral broadening is maximum when the pulses overlap at the center of the fiber. Such precise information on the spectral phase of the pulse propagated in a fiber is very important for the wavelength conversion with spectral broadening or the generation of a monocycle pulse using a spatial light modulator (SLM) that can compensate the spectral phase of a superbroadened continuum. The shortest pulse attainable by phase correction of this ultrabroad spectrum is obtained by the Fourier transform of the spectrum of Fig. 14 and assuming a constant spectral phase. This yields a transform-limited pulse. Figure 16 shows the temporal profiles of the transform-limited pulses obtained numerically for the mixed 
two-pulse spectra shown in Fig. 14 at the center wavelengths of 795 and $640 \mathrm{~nm}$ after simultaneous copropagation with an initial delay of (a) 0 , (b) -40 , and (c) $-80 \mathrm{fs}$ in a $3 \mathrm{~mm}$ silica fiber. The FWHM pulse widths are (a) 4.1, (b) 4.0, and (c) 4.7 fs. The pulse width under an initial delay of $-40 \mathrm{fs}$ is $4.0 \mathrm{fs}$, which is the same value, $4 \mathrm{fs}$ (Xu et al., 1999), obtained by Fourier transform of the experimental result of Fig. 15(b) reported by Xu et al.
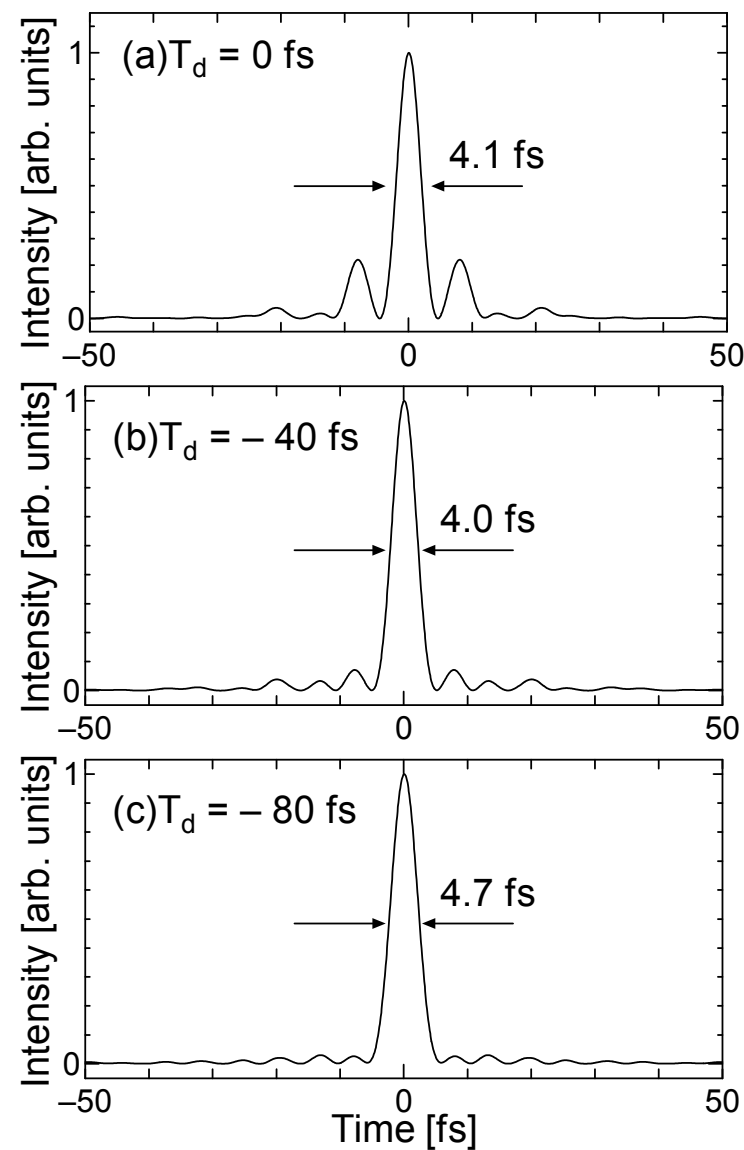

Fig. 16. Temporal profiles of transform-limited pulses obtained numerically for mixed twopulse spectra shown in Fig. 6 at center wavelengths of 795 and $640 \mathrm{~nm}$ after simultaneous copropagation with initial delay of (a) 0 , (b) -40 , and (c) $-80 \mathrm{fs}$ in a $3 \mathrm{~mm}$ silica fiber.

(Nakamura et al., 2005b)

\subsection{Summary of dual wavelengths femtosecond pulses propagation}

The ultrabroad spectrum generation based on the IPM of two optical pulses copropagating in a single-mode fused-silica fiber has been numerically demonstrated, which is compared with our previous experimental results. To the best of our knowledge, this is the first comparison between the extended FDTD calculation and the experiment. In the extended FDTD, it was found that the spectral phases of the two pulses around the wavelength of 710 
nm are continuously connected at a phase value of $\phi(\lambda)=10-20 \mathrm{rad}$ without any discrete point. This fact was unknown when BPM was used previously. In the extended FDTD simulation, two $120 \mathrm{fs}$ pulses with wavelengths at 640 and $795 \mathrm{~nm}$ were coupled into a $3 \mathrm{~mm}$ single-mode fiber. At a pulse energy of $20 \mathrm{~nJ}$ coupled into the fiber for both pulses, an ultrabroad coherent spectrum induced by IPM which covered the range from 567 to $897 \mathrm{~nm}$ was obtained and was compared with our previous experiment. This result generally agrees well with the previous experimental results at the optimum initial delay of $-40 \mathrm{fs}$, which corresponds to the situation in which two pulses meet at the center of the fiber, where the spectral width is $\Delta \lambda_{1 / 100}=320 \mathrm{~nm}$. The Fourier transform of the spectrum can yield a $4.0 \mathrm{fs}$ transform-limited pulse that is the same pulse width of the Fourier-transform-limited pulse of the previous experimental results. It opens a path for generating a single-cycle highenergy optical pulse in the near future. It is necessary to use not only fundamental $(795 \mathrm{~nm})$ and signal $(640 \mathrm{~nm})$ pulses, but also the idler pulse $(1067 \mathrm{~nm})$ of the OPA in order to obtain a single-cycle (2.66 fs at the center wavelength of $795 \mathrm{~nm}$ ), which means three-color pulse propagation in a fiber.

Finally, we obtained the spectral phase after fiber propagation with the calculation. The extended FDTD method clarified how the two pulse phases maintain their connection with each other because it includes no assumption for the two-pulse case, which implies that the method can be used to calculate two different pulses simultaneously with three delays. We reconfirmed that the spectral phases of two different pulses are connected continuously in any case of three initial delays. We found in the IPM spectral phase that the DFWM occurs with an initial delay of $-80 \mathrm{fs}$, which corresponds to the situation in which two pulses meet at the end of the fiber. To the best of our knowledge, this is the first simultaneous observation of DFWM and IPM by FDTD simulation.

\section{Slowly varying envelope approximation breakdown in fiber propagation}

\subsection{Slowly varying envelope approximation breakdown}

There has recently been significant interest in the generation of single-cycle optical pulses by optical pulse compression of ultrabroad-band light produced in fibers. There have been some experiments reported on ultrabroad-band-pulse generation using a silica fiber (Nakamura et al., 2002a), (Karasawa et al., 2000) and an Ar-gas-filled hollow fiber (Karasawa et al., 2001), and optical pulse compression by nonlinear chirp compensation (Nakamura et al., 2002a), (Karasawa et al., 2001) . For these experiments on generating few-optical-cycle pulses, characterizing the spectral phase of ultrabroad-band pulses analytically as well as experimentally is highly important.

Conventionally, the slowly varying envelope approximation (SVEA) in the beam propagation method (BPM) has been used to describe the propagation of an optical pulse in a fiber. (Agrawal, 1995) However, if the pulse duration approaches that of the optical cycle regime $(<10 \mathrm{fs}$ ), this approximation becomes invalid. (Agrawal, 1995) It is necessary to use the finite-difference time-domain (FDTD) method (Joseph \& Taflove, 1997), (Kalosha \& Herrmann, 2000) without SVEA. (Agrawal, 1995) In previous reports, Goorjian and coworkers (Goorjian et al., 1992), (Joseph et al., 1993)), Joseph and coworkers (Joseph \& Taflove, 1997) (Goorjian et al., 1992)' (Joseph et al., 1993)), Taflove and coworkers (Joseph \& Taflove, 1997) (Goorjian et al., 1992)-(Taflove \& Hagness, 2000) and Hagness and coworkers (Goorjian et al., 1992), (Taflove \& Hagness, 2000), (JGTH) proposed an excellent FDTD algorithm considering a combination of linear dispersion with one resonant frequency and nonlinear terms with a Raman response function. 
We performed an experiment of $12 \mathrm{fs}$ optical pulse propagation, as described in $\S 4$. In order to compare FDTD calculation results with the experimentally measured ultrabroad-band spectra of such an ultrashort laser pulse, we extend the JGTH algorithm to that considering all of the exact Sellmeier fitting values for ultrabroad-band spectra. Because of the broad spectrum of pulses propagating in the fiber, it becomes much more important to take an accurate linear dispersion into account. It is well known that at least two resonant frequencies are required for the linear dispersion to accurately fit the refractive index data. In a recent report, Kalosha and Herrmann considered the linear dispersion with two resonant frequencies and the nonlinear terms without the Raman effect.(Kalosha \& Herrmann, 2000). For the single-cycle pulse-generation experiment, we must use at least the shortest pulse of 3.4 fs (Yamane et al., 2003) or sub-5 fs (Karasawa et al., 2001), (Cheng et al., 1998) or $7.1 \mathrm{fs}$ (Nakamura et al., 2002a) or the commercially available $12 \mathrm{fs}$ pulses. Such a time regime is comparable to the Raman characteristic time of $5 \mathrm{fs}$ (Agrawal, 1995) in a silica fiber. Therefore, it is very important to consider not only an accurate linear dispersion of silica but also the Raman effect in a silica fiber in the few-optical-cycles regime. In addition, because of the high repetition rate and pulse intensity stability in particular, ultrabroadband supercontinuum light generation and few-optical-cycles pulse generation by nonlinear pulse propagation in photonic crystal fibers (Ranka et al., 2000) and tapered fibers (Birks et al., 2000), which are both made of silica, have attracted much attention. We have extended the FDTD method, (Nakamura et al., 2002b) with nonlinear polarization $P_{\mathrm{NL}}$ involving the Raman response function (JGTH-algorithm) to $12 \mathrm{fs}$ ultrabroad-band-pulse propagation in a silica fiber with the consideration of linear polarization $P_{\mathrm{L}}$, including all exact Sellmeier-fitting values of silica with three resonant frequencies, in order to compare the calculation results with our experimental results(Nakamura et al., 2002a), (Karasawa et al., 2000b). In this section, we describe the details of the calculation algorithm of the extended FDTD method (Nakamura et al., 2002b) and we also compare the extended FDTD method (Nakamura et al., 2002b) with BPM by applying the split-step Fourier (SSF) method, which is the solution of a modified generalized nonlinear Schrödinger equation (MGNLSE) (Sone et al., 2002), with SVEA, precisely considering the same Raman response function as that of the extended FDTD method, and up to the fifth-order dispersion. Then, in the calculations, the pulse width is gradually shortened from $12 \mathrm{fs}$ to $7 \mathrm{fs}$ to $4 \mathrm{fs}$. Moreover, the soliton number $N$ is established as 1 or 2. To the best of our knowledge, this is the first observation of the breakdown of SVEA as a function of the laser pulse width and soliton number by comparison between the extended FDTD (Nakamura et al., 2002b) and BPM calculations for the nonlinear propagation of a very short $(<12 \mathrm{fs})$ laser pulse in a silica fiber.

\subsection{Beam propagation method}

Conventionally, BPM for solving the generalized nonlinear Schrödinger equation (GNLSE) including SVEA has been used to describe the ultrashort-laser-pulse propagation in an optical fiber. The GNLSE is expressed as

$$
\begin{aligned}
\frac{\partial A}{\partial z}= & -\frac{j}{2 !} \beta_{2} \frac{\partial^{2} A}{\partial T^{2}}+\frac{1}{3 !} \beta_{3} \frac{\partial^{3} A}{\partial T^{3}} \\
& -\frac{\alpha_{\mathrm{a}}}{2} A+j N^{2}\left[|A|^{2} A+\frac{j}{\omega_{0}} \frac{\partial}{\partial T}\left(|A|^{2} A\right)-T_{\mathrm{R}} A \frac{\partial|A|^{2}}{\partial T}\right]^{\prime}
\end{aligned}
$$




$$
N^{2}=\gamma P_{0} T_{0}^{2} /\left|\beta_{2}\right|, \gamma=n_{2} \omega_{0} /\left(c A_{\text {eff }}\right),
$$

where $A$ is the slowly varying amplitude of the pulse envelope of an electric field, which is normalized by $N, \alpha_{\mathrm{a}}$ is the absorption coefficient, $j$ is an imaginary unit, $T_{0}$ is the incident pulse width which is expressed using the full width at half maximum (FWHM) pulse width $t_{\mathrm{p}}$ as $T_{0}=t_{\mathrm{p}} /(2 \sqrt{\ln 2})$ for a Gaussian pulse and $T_{0}=t_{\mathrm{p}} /\{2 \ln (1+\sqrt{2})\}$ for a sech ${ }^{2}$ pulse, $c$ is the velocity of light in vacuum, $\omega_{0}$ is the center angular frequency of the incident pulse, $n_{2}$ is the nonlinear refractive index $\left(\mathrm{m}^{2} / \mathrm{W}\right), P_{0}$ is the incident pulse peak power, $A_{\text {eff }}$ is the effective core area, $T_{\mathrm{R}}$ is the Raman time constant and $T_{\mathrm{R}}=5$ fs. (Agrawal, 1995) $\beta_{n}$ $(n=1,2,3, \cdots)$ is the $n$-th-order derivative of the propagation constant $\beta_{0}$ at an incident center angular frequency $\omega_{0}$ is as follows:

$$
\beta_{n}=\left(\frac{d^{n} \beta}{d \omega^{n}}\right)_{\omega=\omega_{0}} .
$$

$T$ is defined as

$$
T=t-\frac{z}{v_{\mathrm{g}}}=t-\beta_{1} z
$$

where $v_{\mathrm{g}}$ is the group velocity of the optical pulse, $t$ is time and $z$ is the distance from the incident edge of a fiber. In our previous letter (Nakamura et al., 2002b), we also used this GNLSE for comparison with the extended FDTD method. However, a linear approximation of the actual Raman gain curve using the Raman time constant of $T_{R}=5 \mathrm{fs}$ (Agrawal, 1995), which is related to the slope of the Raman gain, and a dispersion approximation up to only the third-order dispersion terms are included in GNLSE. In this section, we use a more precise version of the nonlinear Schrödinger equation, MGNLSE (Sone et al., 2002), (Gross \& Manassah, 1992), that includes a Raman response function which is also found in the extended FDTD and up to fifth-order dispersion as follows:

$$
\begin{aligned}
\frac{\partial A}{\partial z}= & -\frac{j}{2 !} \beta_{2} \frac{\partial^{2} A}{\partial T^{2}}+\frac{1}{3 !} \beta_{3} \frac{\partial^{3} A}{\partial T^{3}}+\frac{j}{4 !} \beta_{4} \frac{\partial^{4} A}{\partial T^{4}}-\frac{1}{5 !} \beta_{5} \frac{\partial^{5} A}{\partial T^{5}} \\
& -\frac{\alpha_{a}}{2} A+j N^{2}\left[|A|^{2} A+\frac{j}{\omega_{0}} \frac{\partial}{\partial T}\left(|A|^{2} A\right)+\mathfrak{J}^{-1}\left[\chi_{\mathrm{R}} \mathfrak{J}\left[|A|^{2}\right]\right] A\right]^{\prime}
\end{aligned}
$$

where the symbols $\mathfrak{I}$ and $\mathfrak{J}^{-1}$ are the operators of Fourier transformation and inverse Fourier transformation, and $\chi_{\mathrm{R}}$ is the generalized Raman-scattering susceptibility. The generalized Raman-scattering susceptibility (Gross \& Manassah, 1992) can be approximated in the harmonic oscillator model for the molecular vibrations as

$$
\chi_{\mathrm{R}}(\Omega)=\frac{\chi_{0}\left(\Omega_{\mathrm{R}} \Gamma_{\mathrm{R}}\right)}{\Omega_{\mathrm{R}}{ }^{2}-\Omega-j \Gamma_{\mathrm{R}} \Omega_{\mathrm{R}}},
$$

where $\Omega=\left(\omega-\omega_{0}\right) T_{0}$ is the angular frequency normalized to the pulse width, and $\Omega_{\mathrm{R}}\left(=13.2 \mathrm{THz} \times 2 \pi \mathrm{T}_{0}\right)$ (François, 1991) is the Raman shift normalized to the pulse width, 
i.e., the molecular vibrational frequency multiplied by $T_{0}$, and $\Gamma_{\mathrm{R}}\left(=15.44 \mathrm{THz} \times 2 \pi T_{0}\right)$ (François, 1991) is the normalized phenomenological linewidth.

\subsection{Experiment}

Figure 1 shows the setup used for the our experiments. (Nakamura et al., 2002a), (Karasawa, et al., 2000). The $12 \mathrm{fs}$ and $10 \mathrm{~nJ}$ pulses $\left(\mathrm{sech}^{2}\right)$ at the center wavelength of $800 \mathrm{~nm}$ were generated from a mode-locked Ti:sapphire laser (Femtolaser $\mathrm{GmbH}$, Femtosource M-1). The incident pulse width was measured by a fringe-resolved autocorrelator (FRAC). The $12 \mathrm{fs}$ pulses were coupled into a $2.5 \mathrm{~mm}$ silica fiber by a 36x reflective objective (Ealing). The advantage of this kind of reflective objective is that no additional group-delay dispersion (GDD) or third-order dispersion (TOD) is introduced to the pulses. The peak power of the input pulse was $175 \mathrm{~kW}$. A single-mode fused silica fiber (Newport F-SPV) with a core diameter of $2.64 \mu \mathrm{m}$ was used. The output from the $2.5 \mathrm{~mm}$ fiber was collimated by another reflective objective and measured by a spectrometer (Ocean Optics, S-2000). The obtained input and output spectra of the fiber are shown in Fig. 2.

\subsection{Numerical results}

\section{A. Comparison between experimental and numerical results}

In our calculations for a fused silica fiber, the parameters in eq. (10) are set as $b_{1}=0.6961663$, $b_{2}=0.4079426, b_{3}=0.8974794, \lambda_{1}=0.0684043 \mu \mathrm{m}, \lambda_{2}=0.1162414 \mu \mathrm{m}$, and $\lambda_{3}=9.896161 \mu \mathrm{m}$ (Agrawal, 1995), where $\lambda_{i}=2 \pi c / \omega_{i}$ and $c$ is the velocity of light in vacuum. We use the value of the nonlinear refractive coefficient $n_{2}=2.48 \times 10^{-20} \mathrm{~m}^{2} / \mathrm{W}$ from ref. (Taylor et al., 1996), and the third-order susceptibility $\chi^{(3)}$ is found to be $\chi^{(3)}=1.85 \times 10^{-22} \mathrm{~m}^{2} / \mathrm{V}^{2}$ at $800 \mathrm{~nm}$, as given by $\chi^{(3)}=(4 / 3) \varepsilon_{0} c n\left(\omega_{0}\right)^{2} n_{2}$, where $\omega_{0}$ is the center angular frequency of the optical pulse. The parameters $\alpha, \tau_{1}$, and $\tau_{2}$ in Eq. (15) are set to be $\alpha=0.7, \tau_{1}=12.2 \mathrm{fs}$ and $\tau_{2}=32 \mathrm{fs}$ (Agrawal, 1995). A single time step of the finite difference is set as $\Delta t=4.4475215 \times 10^{-17} \mathrm{~s}$ at the wavelength of $800 \mathrm{~nm}$. The time step of $\Delta t$ at $800 \mathrm{~nm}$ is defined as the optical cycle at $800 \mathrm{~nm}$ of $2.6666667 \mathrm{fs}$ divided by 60 . The time step for the wavelength of $1550 \mathrm{~nm}$ is defined by the same rule as described above.

In the extended FDTD calculation, we set all parameters to be the same as those in our experiment (Nakamura et al., 2002a), (Karasawa et al., 2000). We compare the results of the extended FDTD calculation and the SSF calculation with the experimental result for the pulse peak power of $175 \mathrm{~kW}$ in order to generate an ultrabroad spectrum which can finally be compressed to $7.1 \mathrm{fs}$ (Nakamura et al., 2002a) or shorter. The total fiber length of $L=2.5$ mm corresponds to 136,500 spatial steps, which means that $L=136,500 \times \Delta z$, where $\Delta z$ is a unit spatial step in the $z$ direction. We need 293,000 time steps to measure the electric field up to the complete passage of the pulse tail. The peak power of the input pulse is set to be $175 \mathrm{~kW}$ (soliton number $N=2.09$ ). The initial temporal pulse form is assumed to be Fouriertransformed sech ${ }^{2}$ because the mode-locked pulse generally has the sech ${ }^{2}$ shape. Hence the input spectrum is naturally assumed to have a sech ${ }^{2}$ shape. The initial pulse width is $12 \mathrm{fs}$ (FWHM). The effective core area $A_{\text {eff }}$ is set to be $5.47 \mu \mathrm{m}^{2}$.

Figure 17(a) shows the results calculated by the extended FDTD Maxwell equation method (A), the solution of MGNLSE obtained using the SSF method with SVEA (B) (up to the fifthorder dispersion terms with the Raman term using the Raman response function), and our previously reported experimental result (Karasawa et al., 2000) (C). It is seen that with 

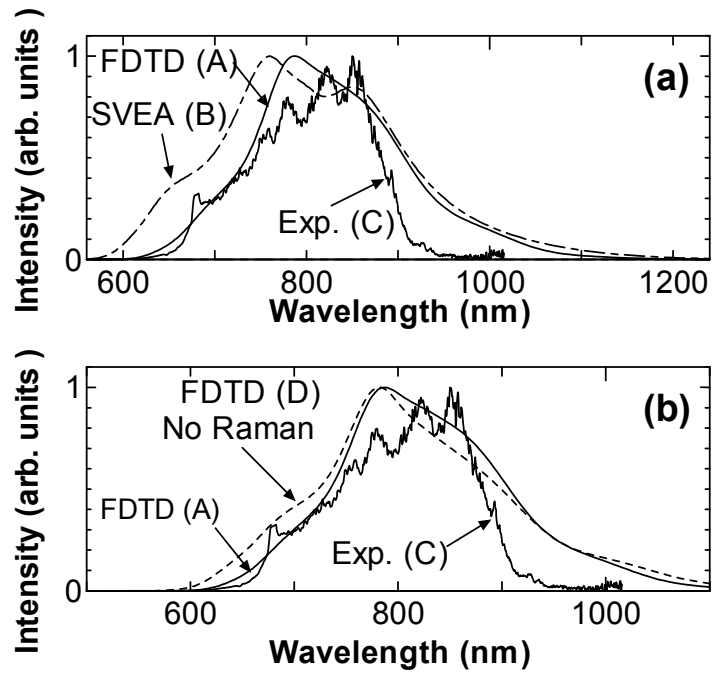

Fig. 17. (a) Spectra of $12 \mathrm{fs}$ laser transmission through a $2.5 \mathrm{~mm}$ silica fiber calculated using (A) the extended FDTD Maxwell equation considering all orders of dispersions and the Raman response [ $\alpha=0.7$ in eq. (15)] and (B) the solution of MGNLSE obtained using the SSF method with SVEA (considering up to 5th-order dispersion terms and the Raman term using the Raman response function), and (C) our previously reported experimental result (Karasawa et al., 2000), where the incident laser intensity corresponds to the soliton number of 2.09. (b) Spectra calculated by the FDTD Maxwell equation method (D) without the Raman response [ $\alpha=1$ in eq. (15)] and (A) with the Raman response. (A) and (C) are the same as those in (a). (Nakamura et al., 2004)

SVEA (B), the spectral intensity at short wavelengths and at long wavelengths is much higher and slightly higher than those for FDTD (A) and the experimental result (C), respectively. The shortest wavelengths (intensity of 1\%) of the spectra of FDTD (A), SVBA (B) and the experimental result (C) are $600 \mathrm{~nm}, 560 \mathrm{~nm}$ and $600 \mathrm{~nm}$, respectively. The longest wavelengths of FDTD (A), SVBA (B) and the experimental result (C) are $1160 \mathrm{~nm}$, $1240 \mathrm{~nm}$ and $1016 \mathrm{~nm}$ (maximum measurable wavelength of spectrometer), respectively. The FWHM spectral bandwidths $\Delta \lambda$ of FDTD (A), SVBA (B) and the experimental result (C) are $172 \mathrm{~nm}, 214 \mathrm{~nm}$ and $136 \mathrm{~nm}$, respectively. Thus the spectral bandwidth of the experimental result (C) is narrow and that of FDTD (A) is closer to the experimental result (C) than is that of SVEA (B). This indicates that the extended FDTD directly solving Maxwell equation is superior to BPM in which MGNLSE (Sone et al., 2002) is solved by SSF with SVEA. The Raman response function (Gross et al., 1992), which is also included in the extended FDTD, and up to the fifth-order dispersion terms are accurately included in BPM for solving MGNLSE (Sone et al., 2002) by SSF with SVEA (B). However, in BPM for solving MGNLSE by SSF with SVEA, the second derivative of the electric field with respect to $z$, $\partial^{2} E_{y} / \partial z^{2}$, is neglected, which corresponds to neglecting the backward-propagating wave. On the other hand, our extended FDTD Maxwell equation method (A) accurately includes the delayed Raman response and all orders of dispersion in silica using Sellmeier's equation, 
and does not require SVEA. Thus, the difference between (A) and (B) is considered to be due to the higher order (more than 6th order) dispersion effect, or the backward-propagating wave. For wavelengths longer than $800 \mathrm{~nm}$ in Fig. 17(a), the extended FDTD result and the $\mathrm{BPM}$ result are similar but the intensity of the experimental data is lower than those of both calculated results. We assume the sensitivity of the spectrometer detector to be low at wavelengths longer than $800 \mathrm{~nm}$ because the detector is made of silicon. We believe our extended FDTD result is accurate. We suggest that it is better to use an IR detector made of a material such as InGaAs for the spectrometer.

Next, in order to clarify the importance of the Raman response, we performed a calculation using (D) the FDTD Maxwell equation method without the Raman response $[\alpha=1$ in eq. (15)], as shown in Fig. 17(b), where (A) and (C) show the same data as those in Fig. 1(a). In Fig. 17(b), the spectrum for case (A) is closer to the experimental result $(\mathrm{C})$ than that of the case of FDTD in which the Raman effect is not considered (D). It is evident that by including the Raman term (A), the spectral intensity at a shorter wavelength is lower, and the agreement between the experimental and calculated results becomes better than that in the case of (D). For example, the spectral intensity at $700 \mathrm{~nm}$ in (D) is $48 \%$ higher than that in (A), which is almost the same as that in the experimental result (C). On the other hand, at a longer wavelength, for example, $850 \mathrm{~nm}$, the spectral intensity of (A) is $15 \%$ higher than that of (D). This feature of (A) shows a tendency analogous to that of (C) because there is a higher peak at $850 \mathrm{~nm}$ than at the center wavelength of $800 \mathrm{~nm}$ in (C). These tendencies of the spectral characteristics indicate that it is important to include the Raman term.

\section{B. Observations of breakdown of SVEA}

We calculate the time profiles and spectra in silica fibers with lengths of 0 to $2 L_{D}$, where $L_{D}$ is the dispersion length, with Gaussian input pulses of $12 \mathrm{fs}, 7 \mathrm{fs}$ and $4 \mathrm{fs}$ duration (FWHM) at the center wavelengths of $800 \mathrm{~nm}$ and $1550 \mathrm{~nm}$ which simulate the normal group-velocity dispersion (GVD) and anomalous GVD, respectively. The dispersion lengths $L_{\mathrm{D}}$ at $800 \mathrm{~nm}$ and $1550 \mathrm{~nm}$ are $1.4364 \mathrm{~mm}$ and $1.8586 \mathrm{~mm}$ for the $12 \mathrm{fs}$ pulse, respectively. Power regions in this calculation are selected to be sufficiently small to enable comparison with results based on the previous theory (Karasawa et al., 200b), (Agrawal, 1995), which means that we normalize the peak power of the input pulse by a soliton parameter of $N=1$.

Figures 18(a) and 18(b) show the time profiles of the output pulses from the silica fiber with incident pulse width of $12 \mathrm{fs}$ and fiber lengths of 0 to $2 L_{\mathrm{D}}$, where $L_{\mathrm{D}}$ is the dispersion length (Agrawal, 1995), calculated by the extended FDTD and BPM with SVEA, respectively. The dispersion length $L_{\mathrm{D}}$ for $12 \mathrm{fs}$ pulses at $800 \mathrm{~nm}$ is $1.4364 \mathrm{~mm}$. The pulse widths simply broaden with propagation in the normal dispersion regime, as shown in Figs. 18(a) and 18(b), because both GVD and self-phase modulation (SPM) (Agrawal, 1995) produce upchirp in normal dispersion. Figures 18(c) and 18(d) show the Fourier-transformed spectra of Figs. 18(a) and 18(b), where $v$ and $v_{0}$ are the frequency and the center frequency of the pulse, respectively. The symmetric spectral broadening due to the SPM effect is seen in the normal dispersion regime, as shown in Figs. 18(c) and 18(d).

Figure 19 show the case of the pulse width of $7 \mathrm{fs}$ under the same definitions of (a) to (d) as in Fig. 18, where the dispersion length $L_{\mathrm{D}}$ for $7 \mathrm{fs}$ pulses at $800 \mathrm{~nm}$ is $0.48939 \mathrm{~mm}$. This figure indicates that pulse width broadening by positive GVD and spectral broadening by the SPM effect are greater than those in Fig. 18, and there is no difference between the results calculated using FDTD [Figs. 19(a) and 19(c)] and BPM [Figs. 19(b) and 19(d)]. This means 
that there is no breakdown of SVEA. Figure 20 shows the case of the pulse width of $4 \mathrm{fs}$ under the same definitions of (a) to (d) as in Figs. 18 and 19, where the dispersion length $L_{D}$ for $4 \mathrm{fs}$ pulses at $800 \mathrm{~nm}$ is $0.15980 \mathrm{~mm}$. This figure also indicates that pulse width broadening and spectral broadening effects are greater than those in Figs. 18 and 19, and there is again no difference between the results calculated using FDTD [Figs. 20(a) and 20(c)] and BPM [Figs. 20(b) and 20(d)]. However, focusing on the case of $z=2 L_{D}$ in Fig. 20(a) calculated by the extended FDTD, we see that the main peak is slightly doubled or slightly dipped. On the other hand, only one peak exists in the results of BPM in Fig. 20(b). In this case, a very small difference is found but it need not be taken into account. That is, there is no breakdown of SVEA even in the case of a pulse width of $4 \mathrm{fs}$. It is very useful to know that we can use BPM even for extremely short pulses of $4 \mathrm{fs}$ without breakdown of SVEA in the case of a soliton number of 1 in the normal GVD region.
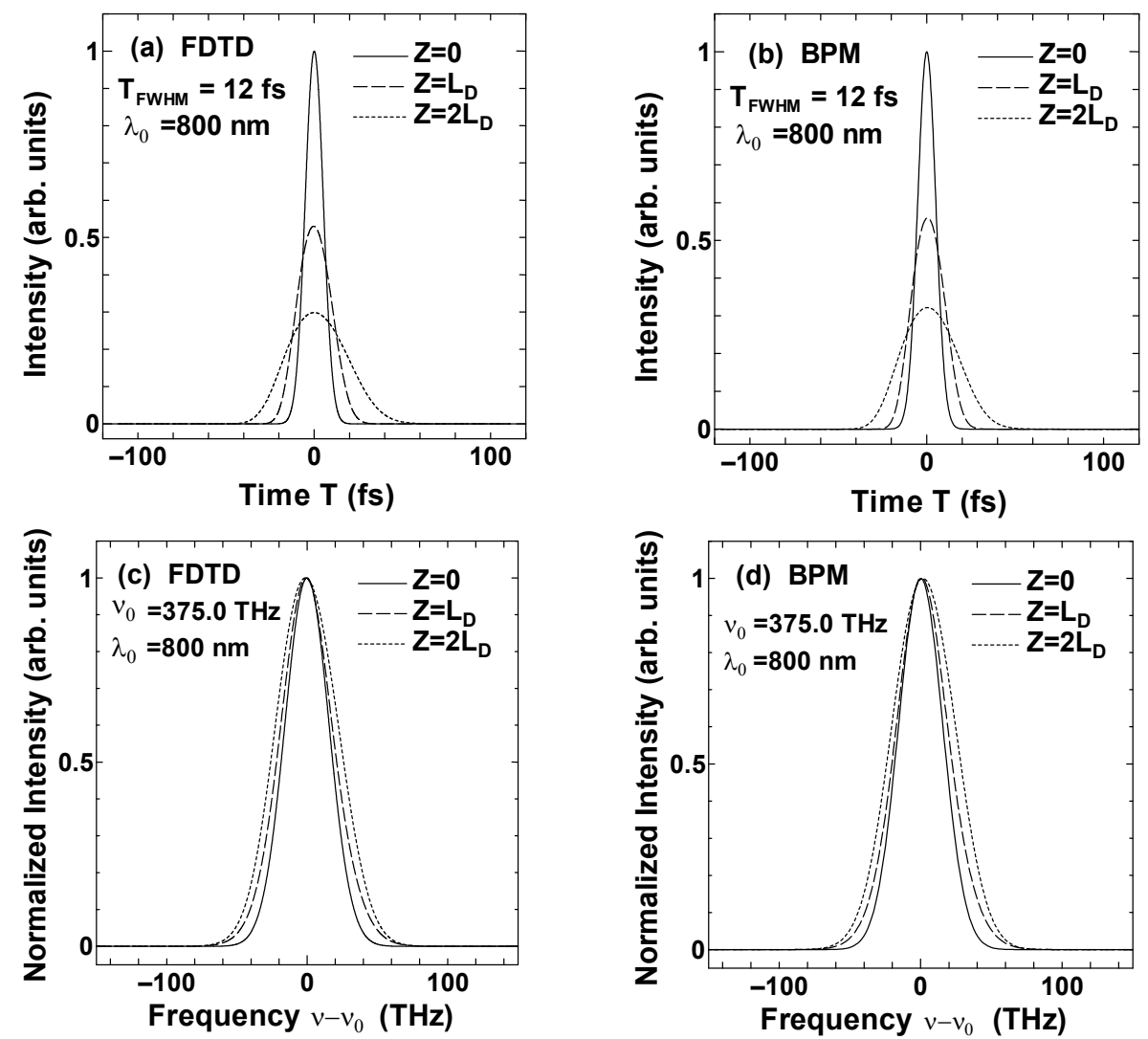

Fig. 18. (a), (b) Temporal profiles and (c), (d) spectra numerically obtained by (a), (c) the extended FDTD method and (b), (d) BPM for $12 \mathrm{fs,} 800 \mathrm{~nm}$ laser propagation through a silica fiber of up to twice the dispersion length $\mathrm{L}_{\mathrm{D}}$, where the laser intensity corresponds to the soliton number of 1 . (Nakamura et al., 2004) 

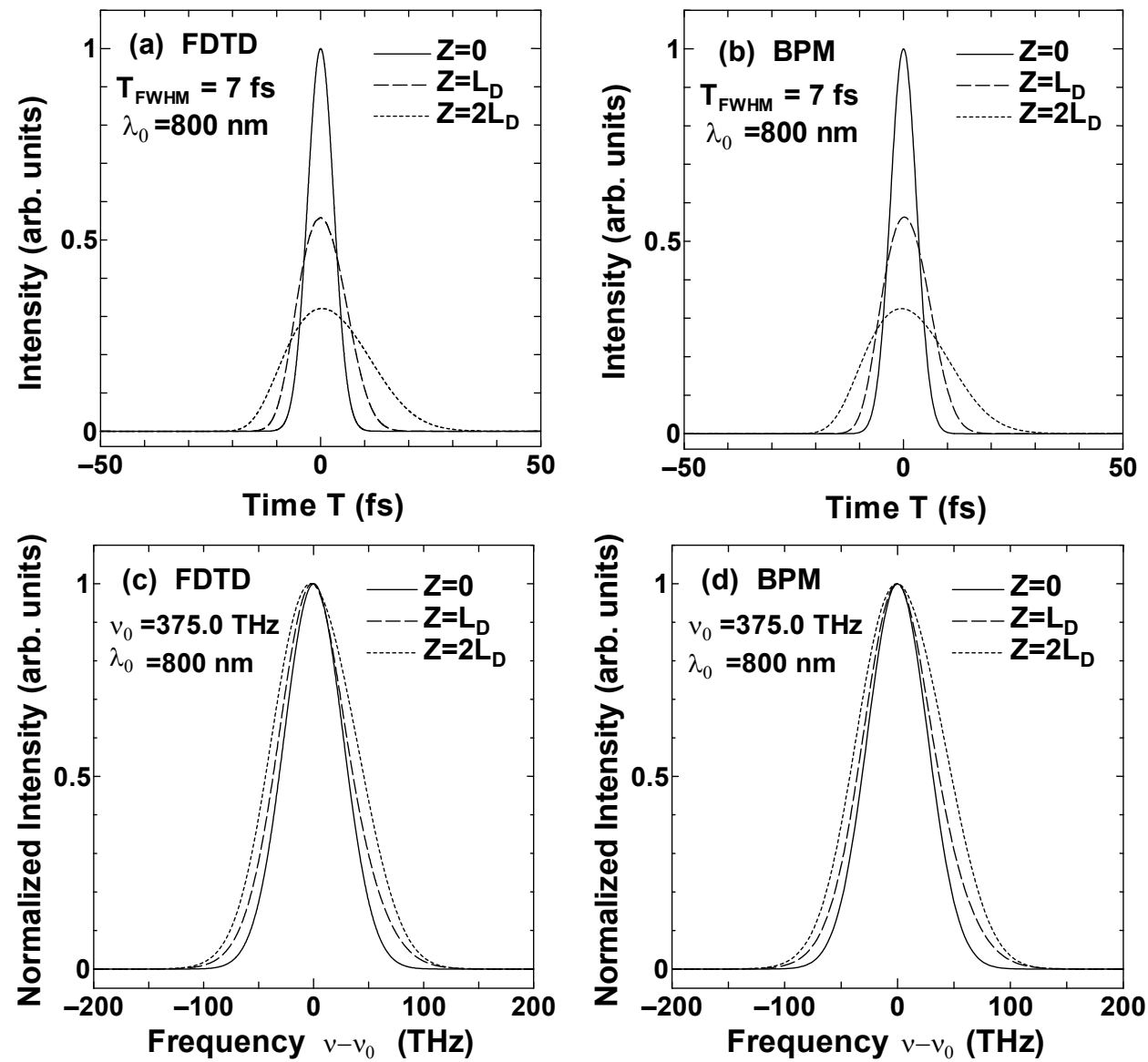

Fig. 19. (a), (b) Temporal profiles and (c), (d) spectra numerically obtained by (a), (c) the extended FDTD method and (b), (d) BPM for $7 \mathrm{fs}, 800 \mathrm{~nm}$ laser propagation through a silica fiber of up to twice the dispersion length $\mathrm{L}_{\mathrm{D}}$, where the laser intensity corresponds to the soliton number of 1. (Nakamura et al., 2004)

On the other hand, in the anomalous dispersion regime, it is seen in Figs. 21(a) and 21(b) that the pulse widths do not vary with pulse evaluation because the soliton effects (Agrawal, 1995) occur as a result of interplay between SPM and anomalous GVD. The dispersion length $L_{\mathrm{D}}$ for $12 \mathrm{fs}$ pulses at $1550 \mathrm{~nm}$ is $1.8586 \mathrm{~mm}$. However, the details of Figs. 21(a) and 21(b) show that the pulse peaks are delayed and appear shifted toward the trailing side with propagation. This is due to self-steepening. (Agrawal, 1995) There are three small peaks following the main peak. These are formed by third-order or higher dispersions. In Figs. 21(a) and 21(b), the second largest peaks slightly differ from each other, and this difference also exists in Figs. 22(a) and 22(b). Figures 21(c) and 21(d) show the Fouriertransformed spectra of Figs. 21(a) and 21(b), where $v$ and $v_{0}$ are the frequency and the center frequency of the pulse, respectively. There is no obvious change of spectral broadening, as shown in Figs. 21(c) and 21(d), because the fundamental soliton is generated, as already described. 

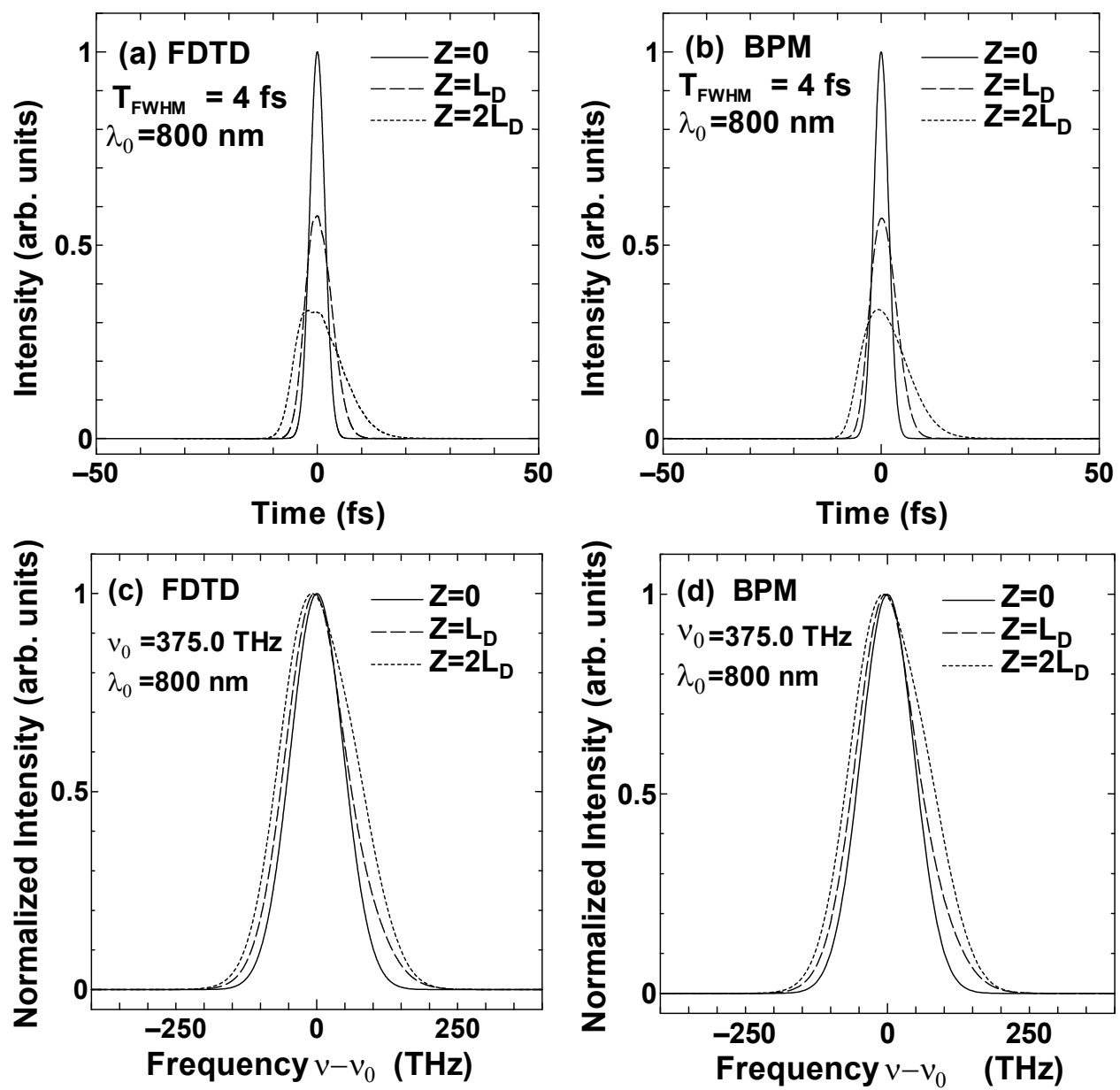

Fig. 20. (a), (b) Temporal profiles and (c), (d) spectra numerically obtained by (a), (c) the extended FDTD method and (b), (d) BPM for $4 \mathrm{fs}, 800 \mathrm{~nm}$ laser propagation through a silica fiber of up to twice the dispersion length $\mathrm{L}_{\mathrm{D}}$, where the laser intensity corresponds to the soliton number of 1. (Nakamura et al., 2004) 

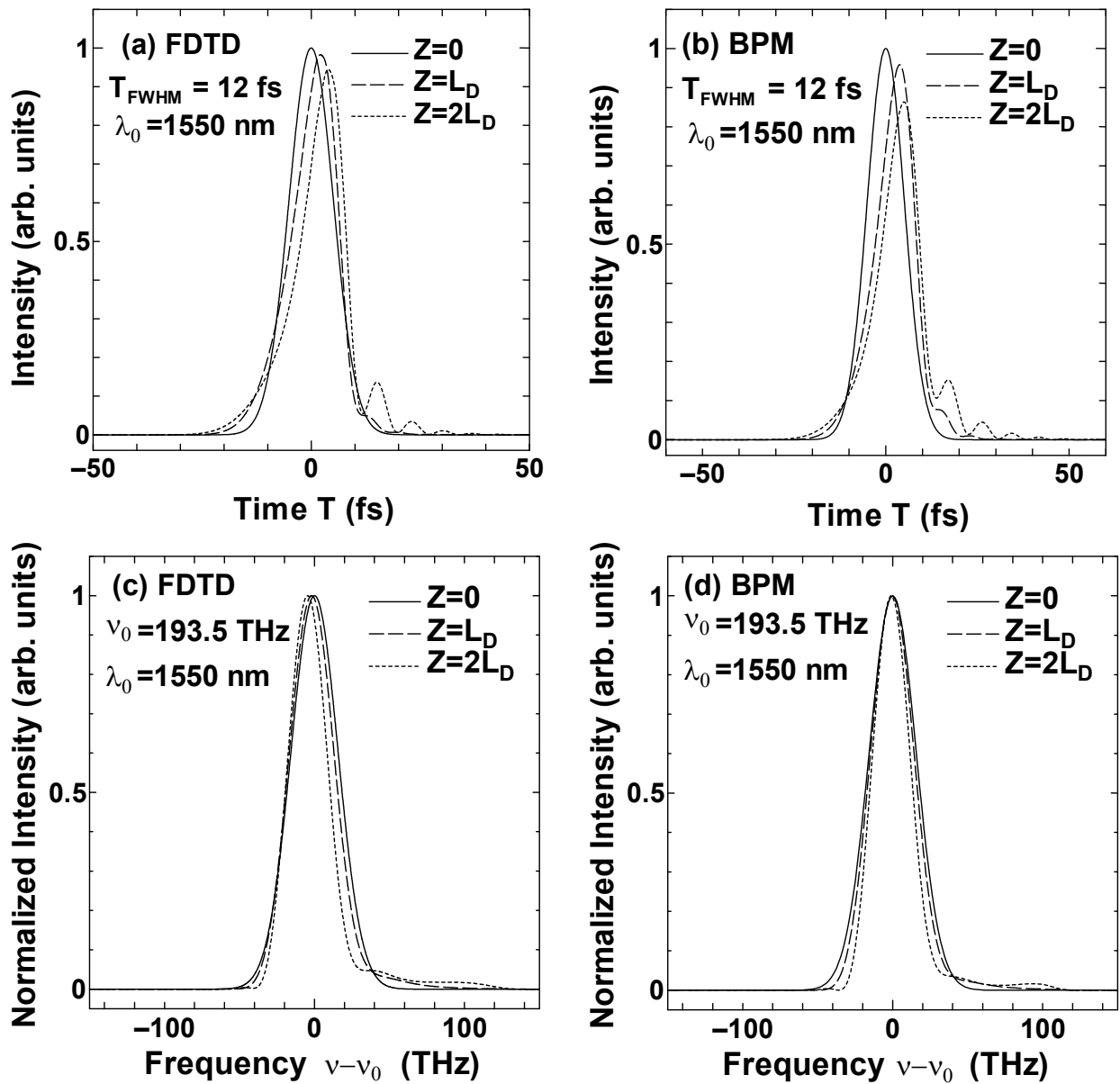

Fig. 21. (a), (b) Temporal profiles and (c), (d) spectra numerically obtained by (a), (c) the extended FDTD method and (b), (d) BPM for $12 \mathrm{fs}, 1550 \mathrm{~nm}$ laser propagation through a silica fiber of up to twice the dispersion length $\mathrm{L}_{\mathrm{D}}$, where the laser intensity corresponds to the soliton number of 1 . (Nakamura et al., 2004) 

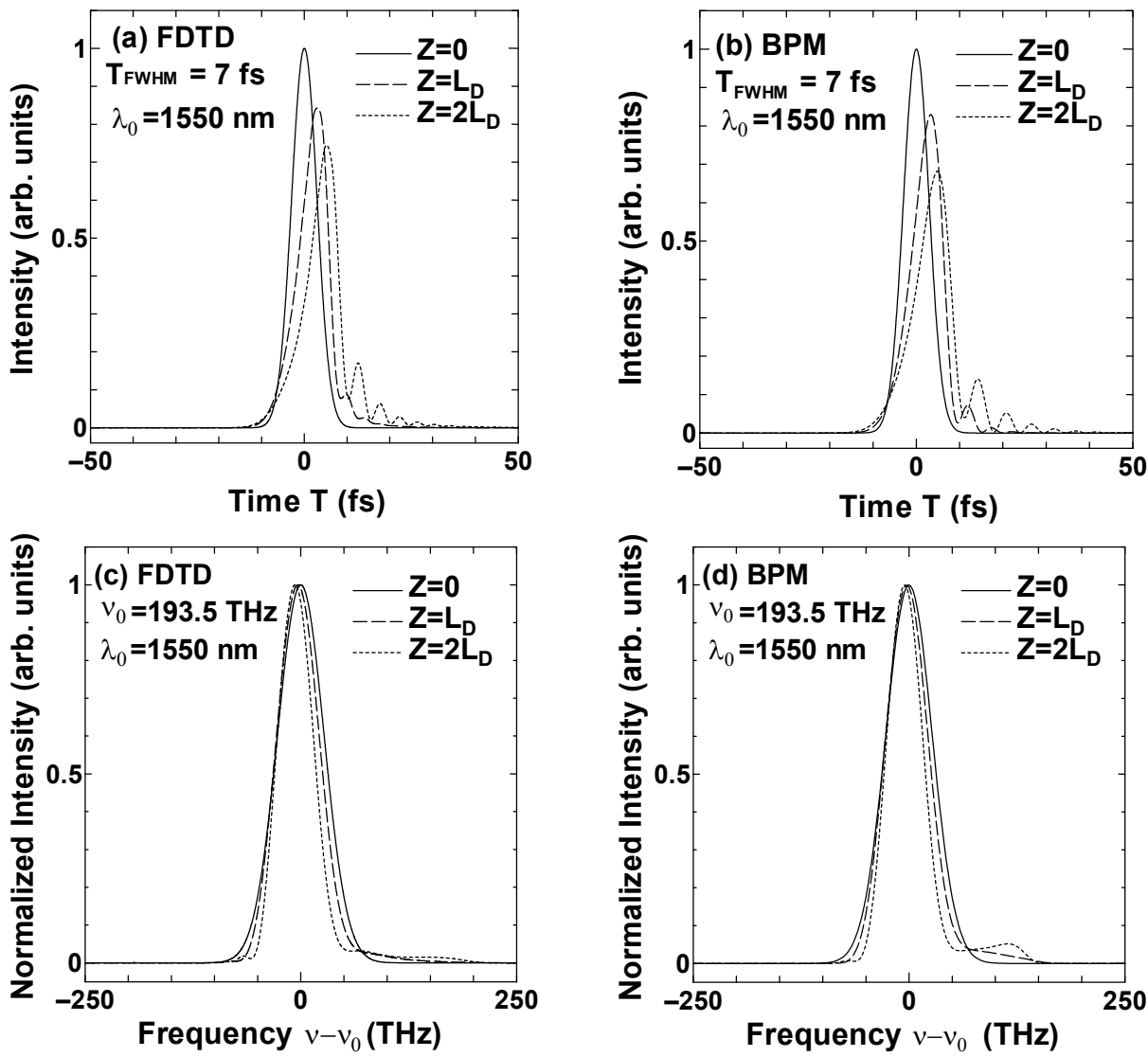

Fig. 22. (a), (b) Temporal profiles and (c), (d) spectra numerically obtained by (a), (c) the extended FDTD method and (b), (d) BPM for $7 \mathrm{fs}, 1550 \mathrm{~nm}$ laser propagation through a silica fiber of up to twice the dispersion length $L_{D}$, where the laser intensity corresponds to the soliton number of 1. (Nakamura et al., 2004)

However, it is seen that there is a small spectral continuum component from $v-v_{0}=20$ to $120 \mathrm{THz}$ in Figs. 22(c) and 22(d). This feature is due to blue shift (Agrawal, 1995) by selfsteepening. This effect becomes more obvious in the case of the pulse widths of $7 \mathrm{fs}$ [Figs. 22(c) and 22(d)] and 4 fs [Figs.23(c) and 23(d)], where the dispersion lengths $L_{D}$ for $7 \mathrm{fs}$ and 4 fs pulses at $1550 \mathrm{~nm}$ are $0.63324 \mathrm{~mm}$ and $0.20677 \mathrm{~mm}$, respectively. We also note that the spectra at $z=2 L_{\mathrm{D}}$ in Figs. 21(c) and 21(d) shift slightly to a lower frequency due to the Raman effect. (Agrawal, 1995) The Raman shift in Fig. 21(c) is larger than that in Fig. 21(d). This Raman effect also become more obvious in the case of the pulse widths of $7 \mathrm{fs}$ [Figs. 22(c) and 22(d)] and 4 fs [Figs.23(c) and 23(d)], and the Raman shift difference between (c) and (d) become smaller with decreasing pulse width. In terms of the Raman effect, FDTD and BPM result in analogous behavior. On the other hand, the behavior of blue shift differs between Fig. 23(c) and Fig. 23(d). The second largest slow perceptible peak exists from $v-v_{0}$ $=100$ to $200 \mathrm{THz}$ in Fig. 23(d), but the frequency region is a small continuum in Fig. 23(c). 
From the total viewpoint of the anomalous region, the breakdown of SVEA is tolerant in 12 $\mathrm{fs}$, however, in the time region shorter than $7 \mathrm{fs}$, the breakdown cannot be ignored.

Through these calculations, we successfully demonstrated the dispersion and SPM effect (Agrawal, 1995), soliton propagation at $1550 \mathrm{~nm}$, self-steepening, and the Raman effect. Furthermore it was found that BPM and the extended FDTD results are almost the same in the normal GVD region, and SVEA does not break down in the cases of incident pulse widths of $12 \mathrm{fs}, 7 \mathrm{fs}$, and $4 \mathrm{fs}$ and soliton number of $N=1$ in the normal dispersion frequency region, as well as in the case of the incident pulse width of $12 \mathrm{fs}$ and soliton number of $N=1$ in the anomalous dispersion frequency region.
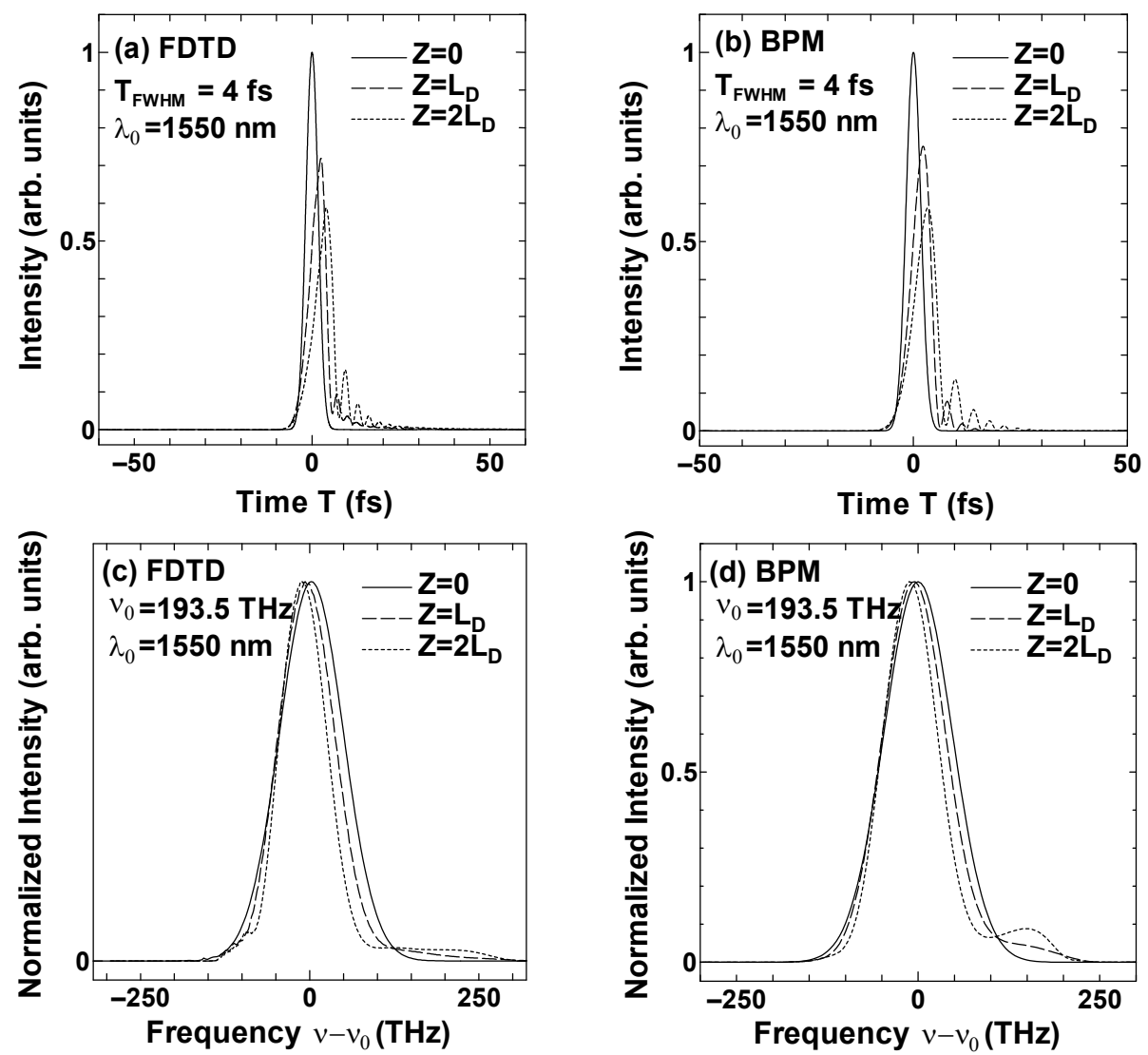

Fig. 23. (a), (b) Temporal profiles and (c), (d) spectra numerically obtained by (a), (c) the extended FDTD method and (b), (d) BPM for $4 \mathrm{fs}, 1550 \mathrm{~nm}$ laser propagation through a silica fiber of up to twice the dispersion length $\mathrm{L}_{\mathrm{D}}$, where the laser intensity corresponds to the soliton number of 1 . (Nakamura et al., 2004) 

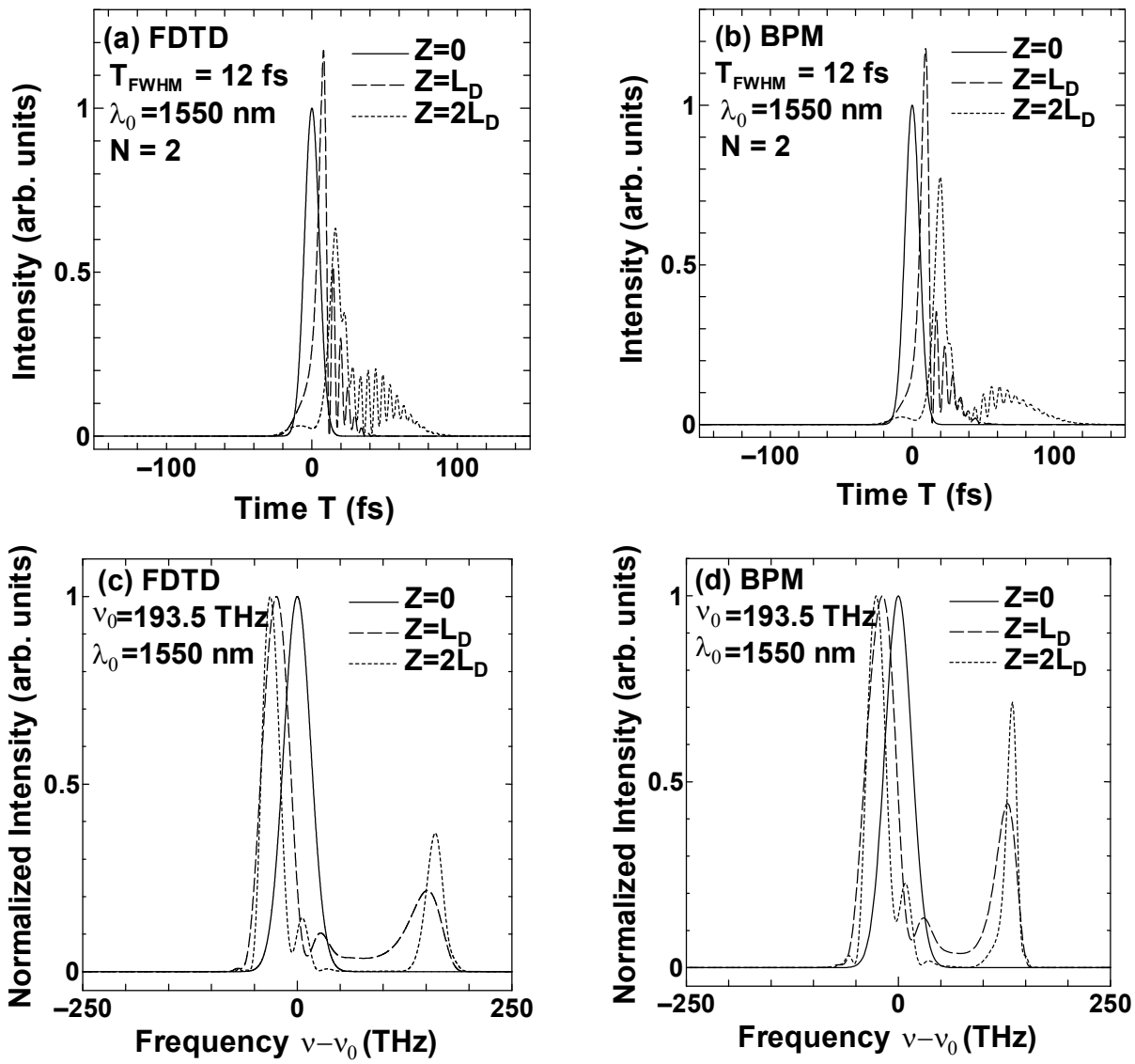

Fig. 24. (a), (b) Temporal profiles and (c), (d) spectra numerically obtained by (a), (c) the extended FDTD method and (b), (d) BPM for $12 \mathrm{fs}, 1550 \mathrm{~nm}$ laser propagation through a silica fiber of up to twice the dispersion length $\mathrm{L}_{\mathrm{D}}$, where the laser intensity corresponds to the soliton number of 2. (Nakamura et al., 2004)

Although we obtained extended FDTD results which could be explained by the previous theory (Agrawal, 1995) and could verify the validity of our calculation method, the physical behavior of very short pulses will be different when the peak power is four times larger than that in the case of $N=1$, which makes the breakdown of SVEA possible even for long pulses. We have already shown the breakdown of SVEA in a comparison of experimental and numerical results for the positive dispersion area with $N=2.09$ for 12 fs pulse propagation in the fiber. Thus, we perform calculations for $N=2$ and the anomalous dispersion area.

Figure 24 shows (a), (b) temporal profiles and (c), (d) spectra numerically obtained by (a), (c) the extended FDTD method and (b), (d) BPM for $12 \mathrm{fs,} 1550 \mathrm{~nm}$ laser propagation through a silica fiber of up to $z=2 L_{\mathrm{D}}$, where the laser intensity corresponds to the soliton number $N$ of 2. This figure shows that the breakdown of SVEA occurs in BPM for even a long pulse width of 12 fs. For the spectra shown in Figs.24(c) and 24(d), the height of the second largest peak 
in Fig. 24(d) is twice that in Fig. 10(c). In the temporal profiles of Figs. 24(a) and 24(b), the main peaks have the same form, however numerous small-peak characteristics from 20 fs to $80 \mathrm{fs}$ are different. The small peaks in Fig. 24(a) are larger and more obvious than those in Fig. 24(b). These results imply that SVEA of MGNLSE in BPM, as assumed in Figs. 24(b) and 24(d), breaks down in the case of $N=2$ in the anomalous GVD region for $12 \mathrm{fs}$ pulse propagation.

\subsection{Summary of SVEA breakdown}

We have extended the nonlinear FDTD method with a JGTH algorithm to one with exact Sellmeier fitting values in order to compare the experimental and calculated results of nonlinear femtosecond ultrabroad-band-pulse propagation in a silica fiber, and have analyzed the spectral characteristics of the propagated pulse. This extended method is robust against the breakdown of the SVEA. The spectrum obtained in our previous experiment agrees better with the spectrum calculated by the extended FDTD method than with that calculated by the solution of MGNLSE obtained using the SSF method with SVEA. Consequently, we confirmed that the various nonlinear physical phenomena in an optical fiber can be described by FDTD methods even in a nearly single-cycle time regime, and that SVEA in BPM is invalid in the case of pulse widths less than $4 \mathrm{fs}$ with the soliton number of 1 at $800 \mathrm{~nm}$, which is the normal dispersion regime, and in the case of pulse widths less than $7 \mathrm{fs}$ with the soliton number of 1 at $1550 \mathrm{~nm}$, which is the anomalous dispersion regime. In the case of the soliton number of 2, the breakdown of SVEA occurs for $12 \mathrm{fs}$ pulses in both the normal and anomalous dispersion frequency regimes. To the best of our knowledge, this is the first observation of the breakdown of SVEA as a function of laser pulse width and soliton number by comparison of the extended FDTD and BPM calculations for the nonlinear propagation of an ultrashort $(<12 \mathrm{fs})$ laser pulse in a silica fiber. If the extended FDTD method can be established as an ultrashort-optical-pulse propagation analysis technique, its application to analyses of the characteristics of an ultrahigh-speed optical switch, the propagation characteristic of optical pulses in a photonic crystal, and the nonlinear propagation in a photonic crystal fiber or a tapered fiber can be expected.

\section{Conclusion}

Conventionally, the BPM for solving the GNLSE including the SVEA has been used to describe the ultrashort laser pulse propagation in an optical fiber. However, if the pulse duration approaches the optical cycle regime $(<10 \mathrm{fs})$, this approximation becomes invalid. Then, it becomes necessary to use the FDTD method for solving the Maxwell equation with the least approximation. In order to both experimentally and numerically investigate nonlinear femtosecond ultrabroad-band-pulse propagation in a silica fiber, we have extended the FDTD calculation of Maxwell's equations with nonlinear terms to that including all exact Sellmeier-fitting values. We have compared the results of this extended FDTD method with experimental results for the nonlinear propagation of a very short (12-fs) chirped laser pulse in a silica fiber. We obtain the fiber output pulse compressed to $7 \mathrm{fs}$ by the simulation of group delay compensation under the assumption of using an SLM. To our knowledge, this is the first comparison between FDTD calculation and experimental results for nonlinear propagation of a very short (12-fs) chirped pulse in a silica fiber. 
Next, experimental results of nonlinear propagation with the IPM effect of two-color $120 \mathrm{fs}$ laser pulses with three different initial delays in a silica fiber were analyzed with FDTD calculations, including SPM, IPM, and DFWM in the fused-silica fiber. Initial delay dependence by the FDTD qualitatively agrees with the previous experimental results. An ultrabroad spectrum induced by IPM mostly agrees with the previous experimental results that had the optimum initial delay. A Fourier transform of this spectrum yields a 4 fs optical pulse, which agrees with the previous experimental results. The spectral phases of two different pulses with three different initial delays are connected continuously. The IPM spectral phase obtained by the extended FDTD shows that the DFWM occurs. To the best of our knowledge, this is the first observation of DFWM with IPM by the FDTD simulation.

Finally, We compared the results of this extended FDTD method with the solution of the BPM that includes the Raman response function, which is the same as in the extended FDTD method, up to fifth-order dispersion with the SVEA, as well as with the experimental results for nonlinear propagation of a 12 fs laser pulse in a silica fiber. Furthermore, in only the calculation, pulse width was gradually shortened from $12 \mathrm{fs}$ to $7 \mathrm{fs}$ to 4 fs to observe the breakdown of the SVEA in detail. Moreover, the soliton number $\mathrm{N}$ was established as 1 or 2. To the best of our knowledge, this is the first observation of the breakdown of the SVEA by comparison between the results of the extended FDTD and the BPM calculations for the nonlinear propagation of an ultrashort $(<12 \mathrm{fs})$ laser pulse in a silica fiber.

\section{References}

Agrawal, G. A. (1995). Nonlinear Fiber Optics 2nd ed., Academic Press, ISBN 978-0123743022, San Diego CA, Chaps. 1 and 2.

Birks, T. A.; Wadsworth, W. J. \& Russell, P. St. J. (2000). Supercontinuum generation in tapered fibers. Opt. Lett., Vol. 25, No. 19, (Oct., 2000) 1415-1417, ISSN 0146-9592.

Cheng, Z.; Tempea,; Brabec, G. T.; Ferencz, K.; Spielman, C. \& Krausz, F. (1998). Generation of Intense Diffraction-Limited White Light and 4-fs Pulses. in Ultrafast Phenomena XI, Elsaesser, T.; Fujimoto, J. G.; Wiersma D. A. \& Zinth, W., 8-10, Springer, ISBN 978-3540654308, Berlin.

Cundiff, S. T.; Collings, B. C.; Boivin, L.; Bergman, M. C.; Knox W. H. \& Evangelides, Jr. S. G. (1999). Propagation of highly chirped pulses in fiber-optic communications systems. IEEE J. Lightwave Technol., Vol. 17, No. 5, (May, 1999) 811-816, ISSN 0733-8724.

François, P. L. (1991). Nonlinear propagation of ultrashort pulses in optical fibers: total field formulation in the frequency domain. J. Opt. Soc. Am. B, Vol. 8, No. 2 (Feb. 1991) 276-293, ISSN 0740-3224.

Fujii, M.; Tahara, M.; Sakagami, I; Freude, W. \& Russer, P. (2004). High-Order FDTD and Auxiliary Defferntial Equation Formulation of Optical Pulse Propagation in 2-D Kerr and Raman Nonlinear Dispersive Media. IEEE Journal of Quantum Electronics, Vol. 40, No. 2, (Feb., 2004) 175-182, ISSN 0018-9197.

Geraghty, D. F.; Lee, R. B.; Verdiell, M.; Ziari, M.; Mathur, A \& Vahala, K. J. (1998). Wavelength conversion by four-wave mixing in semiconductor optical amplifiers. IEEE Photonics Technol. Lett., Vol. 10, No. 1, (Jan., 1998) 69-71, ISSN 1041-1135.

Goorjian, P. M.; Taflove, A.; Joseph, R. M. \& Hagness, S. C. (1992). Computational modeling of femtosecond optical solitons from Maxwell's equations. IEEE J. Quantum. Electron., Vol. 28, No. 10, (Oct. 1992) 2416-2422, ISSN 0018-9197. 
Goorjian, P. M.\& Cundiff, S. T.(2004). Nonlinear effects on the carrier-envelope phase. Opt. Lett., Vol. 29, No. 12, (June, 2004) 1363-1365, ISSN 0146-9592.

Gross, B. \& Manassah J. T. (1992). Supercontinuum in the anomalous group-velocity dispersion region J. Opt. Soc. Am. B, Vol. 9, No. 10, (Oct., 1992) 1813-1818, ISSN 07403224.

Joseph, R. M.; Goorjian, P. M. \& Taflove, A. (1993). Direct time integration of Maxwell's equations in 2-D dielectric waveguides for propagation and scattering of femtosecond electromagnetic solitons. Optics Letters, Vol. 18, No. 7, (Apr., 1993), 491493, ISSN 0146-9592.

Joseph, R. M. \& Taflove, A. (1997). FDTD Maxwell's equations models for nonlinear electrodynamics and optics. IEEE Trans. Antenas Propagat., Vol. 45, No. 3, (Mar., 1997), 364-374, ISSN 0018-926X.

Kalosha, V. P. \& Herrmann, J. (2000). Self-phase modulation and compression of few-opticalcycle pulses. Phys. Rev. A, Vol. 62, No. 1, (June, 2000) R11804.1-R11804.4, ISSN 10502947.

Karasawa, N.; Nakamura, S.; Morita, R.; Shigekawa, H. \& Yamashita, M. (2000) Comparison between theory and experiment of nonlinear propagation for 4.5-cycle optical pulses in a fused-silica fiber. Nonlinear Opt., Vol. 24, (Dec. 2000). 133-138, ISSN $1058-7268$.

Karasawa, N.; Morita, R.; Shigekawa, H. \& Yamashita M. (2000b). Generation of intense ultrabroadband optical pulses by induced phase modulation in an argon-filled single-mode hollow waveguide. Opt. Lett., Vol.25, No. 3, (Feb., 2000) 183-185, ISSN 0146-9592.

Karasawa, N.; Li, L.; Suguro, A.; Shigekawa, H.; Morita, R. \& Yamashita, M. (2001) Optical pulse compression to $5.0 \mathrm{fs}$ using only a spatial light modulator. J. Opt. Soc. Am. B, Vol. 18, No. 11, (Nov., 2001) 1742-1746, ISSN 0740-3224.

Karasawa, N.; Nakamura, S.; Nakagawa, N.; Shibata, M.; Morita, R.; Shigekawa, H.\& Yamashita, M. (2001b). Comparison between theory and experiment of nonlinear propagation for a-few-cycle and ultrabroadband optical pulses in a fused-silica fiber IEEE J. Quantum Electron, Vol. 37, No.3, (Nov. 2001) 398-404, ISSN 0018-9197.

Malitson, I. H. (1965). Interspecimen comparison of the refractive index of fused silica. J. Opt. Soc. Am., Vol. 55, No. 10, (Oct. 1965), 1205-1208, ISSN 0740-3224.

Nakamura, S.; Li, L.; Karasawa, N.; Morita, R.; Shigekawa, H. \& Yamashita, M. (2002a). Measurements of Third-Order Dispersion Effects for Generation of HighRepetition-Rate, Sub-Three-Cycle Transform-Limited Pulses from a Glass Fiber. Jpn. J. Appl. Phys., Vol. 41, No. 3A, (Mar. 2002) 1369-1373, ISSN 0021-4922.

Nakamura, S.; Koyamada, Y.; Yoshida, N.; Karasawa, N.; Sone, H.; Ohtani, M.; Mizuta, Y.; Morita, R.; Shigekawa, H. \& Yamashita M. (2002b). Finite-Difference Time-Domain Calculation with All Parameters of Sellmeier's Fitting Equation for 12-fs Laser Pulse Propagation in a Silica Fiber. IEEE Photon. Technol. Lett., Vol. 14, No.4, (Apr. 2002) 480-482, ISSN 1041-1135.

Nakamura, S.; Takasawa, N.; Saeki, T. \& Koyamada, Y. (2004a). Numerical Analysis of Induced Phase Modulation between Two Ultrashort Pulses in a Silica Fiber by Using the Extended Finite-Difference Time-Domain Method. Opt. Rev., Vol. 11, No. 2, (Mar./ Apr., 2004) 68-71, ISSN 1340-6000. 
Nakamura, S; Saeki, T \& Koyamada, Y. (2004b). Observation of Slowly Varying Envelope Approximation Breakdown by Comparison between the Extended FiniteDifference Time-Domain Method and the Beam Propagation Method for Ultrashort-Laser-Pulse Propagation in a Silica Fiber. Japanese Journal of Applied Physics, Vol. 43, No. 10A, (Oct., 2004) 7015-7025, ISSN 0021-4922.

Nakamura, S; Takasawa, N. \& Koyamada, Y. (2005a). Comparison between Finite-Difference Time-Domain Calculation with All Parameters of Sellmeier's Fitting Equation and Experimental Results for Slightly Chirped 12 fs Laser Pulse Propagation in a Silica Fiber. IEEE Journal of Lightwave Technology, Vol. 23, No. 2, (Feb., 2005) 855-863, ISSN 0733-8724.

Nakamura, S; Takasawa, N. \& Koyamada, Y.; Sone, H.; Xu, L.; Morita, R. \& Yamashita, M. (2005b). Extended Finite Difference Time Domain Analysis of Induced Phase Modulation and Four-Wave Mixing between Two-Color Femtosecond Laser Pulses in a Silica Fiber with Different Initial Delays. Japanese Journal of Applied Physics, Vol. 44, No. 10, (Oct., 2005) 7453-7459, ISSN 0021-4922.

Ranka, J. K.; Windeler, R. S. \& Stentz, A. J. (2000). Visible continuum generation in air-silica microstructure optical fibers with anomalous dispersion at $800 \mathrm{~nm}$. Opt. Lett., Vol. 25, No. 1, (Jan., 2000), 25-27, ISSN 0146-9592.

Shibata, M.; Hirasawa, M.; Nakagawa, N.; Morita, R.; Suguro, A.; Shigekawa, H. \& Yamashita, M. (2002). Experimental demonstration of phase-dispersion compensation for ultra-broadband femtosecond optical pulses generated by induced-phase modulation. Appl. Phys. B, Vol. 74, No. 1, (July, 2002) s291-s294, ISSN 0946-2171.

Sone, H.; Arai, T.; Imai, M. \& Imai Y. (2002). Modal Birefringence Dependent Supercontinuum Due to Cross-Phase Modulation in a DispersionFlattened/Decreasing Fiber. Optical Review, Vol. 9, No. 3, (May/Jun., 2002) 89-93, ISSN 1340-6000.

Taflove, A. \& Hagness, S. C. (2000). Computational Electrodynamics: The Finite-Difference TimeDomain Method, 2nd ed., Artech House, ISBN 978-1580538329, Norwood MA, Chapter 9, Section 9.6 (pp. 398-401).

Taylor, A. J.; Rodriguez, G. R. \& Clement, T. S. (1996). Determination of $n_{2}$ by direct measurement of the optical phase. Opt. Lett., Vol. 21, No. 22, (Nov., 1996), 1812-1814 ISSN 0146-9592.

Xu, L; Karasawa, N.; Nakagawa, N.; Morita, R.; Shigekawa, H. \& Yamashita, M. (1999). Experimental generation of an ultra-broad spectrum based on induced-phase modulation in a single-mode glass fiber. Opt. Commun, Vol. 162, No. 4-6, (Apr., 1999) 256-260, ISSN 0946-2171.

Yamane, K.; Zhang, Z.; Oka, K.; Morita, R.; Yamashita, M. \& Suguro, A. (2003). Optical pulse compression to $3.4 \mathrm{fs}$ in the monocycle region by feedback phase compensation. Opt. Lett., Vol. 28, no. 22, (Nov. 2003) 2258-2260, ISSN 0146-9592.

Yamashita, M; Hirasawa, M.; Nakagawa, N.; Yamamoto, K.; Oka, K.; Morita, R. \& Suguro A. (2004). Pulse compression of white-light continuum generated by induced phase modulation in a conventional glass fiber. J. Opt. Soc. Am. B, Vol. 21, No. 2, (Feb., 2004) 458-462, ISSN 0740-3224.

Yamashita, M.; Sone, H.; Morita, R.; \& Shigekawa, H. (1998). Generation of monocycle-like optical pulses using induced-phase modulation between two-color femtosecond 
pulses with carrier phase locking. IEEE J. Quantum Electron, Vol. 34, No. 11, (Nov., 1998) 2145-2149, ISSN 0018-9197.

Yamashita, M.; Sone, H. \& Morita, R. (1996). Proposal for Generation of a Coherent Pulse Ultra-Broadened from Near-Infrared to Near-Ultraviolet and Its Monocyclization. Jpn. J. Appl. Phys., Vol. 35, (Aug., 1996) L1194-L1197, ISSN 0021-4922. 


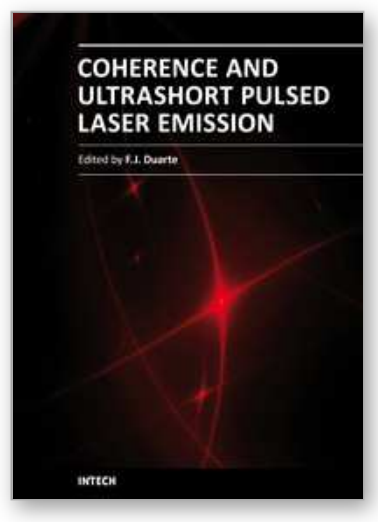

\section{Coherence and Ultrashort Pulse Laser Emission}

Edited by Dr. F. J. Duarte

ISBN 978-953-307-242-5

Hard cover, 688 pages

Publisher InTech

Published online 30, November, 2010

Published in print edition November, 2010

In this volume, recent contributions on coherence provide a useful perspective on the diversity of various coherent sources of emission and coherent related phenomena of current interest. These papers provide a preamble for a larger collection of contributions on ultrashort pulse laser generation and ultrashort pulse laser phenomena. Papers on ultrashort pulse phenomena include works on few cycle pulses, high-power generation, propagation in various media, to various applications of current interest. Undoubtedly, Coherence and Ultrashort Pulse Emission offers a rich and practical perspective on this rapidly evolving field.

\section{How to reference}

In order to correctly reference this scholarly work, feel free to copy and paste the following:

Shinki Nakamura (2010). Comparison between Finite-Difference Time-Domain Method and Experimental Results for Femtosecond Laser Pulse Propagation, Coherence and Ultrashort Pulse Laser Emission, Dr. F. J. Duarte (Ed.), ISBN: 978-953-307-242-5, InTech, Available from: http://www.intechopen.com/books/coherenceand-ultrashort-pulse-laser-emission/comparison-beteween-fdtd-simulation-and-experimental-results-for-12-fslaser-pulse-propagation-in-a-

\section{INTECH}

open science | open minds

\section{InTech Europe}

University Campus STeP Ri

Slavka Krautzeka 83/A

51000 Rijeka, Croatia

Phone: +385 (51) 770447

Fax: +385 (51) 686166

www.intechopen.com

\section{InTech China}

Unit 405, Office Block, Hotel Equatorial Shanghai

No.65, Yan An Road (West), Shanghai, 200040, China 中国上海市延安西路65号上海国际贵都大饭店办公楼 405 单元

Phone: +86-21-62489820

Fax: +86-21-62489821 
(C) 2010 The Author(s). Licensee IntechOpen. This chapter is distributed under the terms of the Creative Commons Attribution-NonCommercialShareAlike-3.0 License, which permits use, distribution and reproduction for non-commercial purposes, provided the original is properly cited and derivative works building on this content are distributed under the same license. 